\title{
Variable Stars and Stellar Populations in Andromeda XXVII. IV. An Off-centered, Disrupted Galaxy*
}

\author{
Felice Cusano ${ }^{1}$ (D) , Alessia Garofalo ${ }^{1,2}$, Gisella Clementini ${ }^{1}$, Michele Cignoni ${ }^{3}$ (D), Tatiana Muraveva ${ }^{1}$, Gianni Tessicini ${ }^{1}$, \\ Vincenzo Testa ${ }^{4}$ (D), Diego Paris ${ }^{4}$, Luciana Federici ${ }^{1}$, Marcella Marconi ${ }^{5}$ (D), Vincenzo Ripepi ${ }^{5}$ (D), and Ilaria Musella ${ }^{5}$ \\ ${ }^{1}$ INAF- Osservatorio Astronomico di Bologna, Via Gobetti 93/3, I-40129 Bologna, Italy; felice.cusano@oabo.inaf.it \\ ${ }^{2}$ Dipartimento di Fisica e Astronomia, Università di Bologna, viale Berti Pichat, 6/2, I-40127 Bologna, Italy \\ ${ }^{3}$ Dipartimento di Fisica, Università di Pisa, Largo Bruno Pontecorvo, 3, I-56127 Pisa PI, Italy \\ ${ }^{4}$ INAF- Osservatorio Astronomico di Roma, Via di Frascati 33, I-00040 Monte Porzio Catone, Italy \\ ${ }^{5}$ INAF- Osservatorio Astronomico di Capodimonte, Salita Moiariello 16, I-80131 Napoli, Italy \\ Received 2017 March 6; revised 2017 October 23; accepted 2017 October 25; published 2017 December 5
}

\begin{abstract}
We present $B$ and $V$ time-series photometry of the M31 satellite galaxy Andromeda XXVII (And XXVII) that we observed with the Large Binocular Cameras of the Large Binocular Telescope. In the field of And XXVII we have discovered a total of 90 variables: 89 RR Lyrae stars and 1 Anomalous Cepheid. The average period of the fundamental mode RR Lyrae stars (RRab) $\left\langle P_{\mathrm{ab}}\right\rangle=0.59$ days $(\sigma=0.05$ day) and the period-amplitude diagram place And XXVII in the class of Oosterhoff I/Intermediate objects. Combining information from the colormagnitude diagram (CMD) and the variable stars, we find evidence for a single old and metal-poor stellar population with $[\mathrm{Fe} / \mathrm{H}] \sim-1.8$ dex and $\mathrm{t} \sim 13 \mathrm{Gyr}$ in And XXVII. The spatial distributions of RR Lyrae and red giant branch (RGB) stars give clear indication that And XXVII is a completely disrupted system. This is also supported by the spread observed along the line of sight in the distance to the RR Lyrae stars. The highest concentration of RGB and RR Lyrae stars is found in a circular area of 4 arcmin in radius, centered about $0^{\circ} .2$ in the southeast direction from Richardson et al.'s center coordinates of And XXVII. The CMD of this region is welldefined, with a prominent RGB and 15 RR Lyrae stars (out of the 18 found in the region) tracing a very tight horizontal branch at $\langle V(R R)\rangle=25.24 \mathrm{mag} \sigma=0.06 \mathrm{mag}$ (average over 15 stars). We show that And XXVII is a strong candidate building block of the M31 halo.
\end{abstract}

Key words: galaxies: dwarf - galaxies: individual (Andromeda XXVII) - Local Group - stars: distances

Supporting material: extended figure

\section{Introduction}

In the $\Lambda$-cold dark matter $(\mathrm{CDM})$ scenario, galaxies are formed by hierarchical assembly of smaller structures (e.g., Bullock \& Johnston 2005; Annibali et al. 2016; Stierwalt et al. 2017). The dwarf satellites of the Andromeda galaxy (M31) can help to constrain the origin and the fate of M31. Through the characterization of the resolved stellar populations and the variable stars in these systems, it is possible to trace the global context of merging and accretion episodes that have occurred and are still occurring in the M31 environment (Martin et al. 2013).

This is the fourth paper in our series on the M31 satellites based on $B$ and $V$ time-series photometry obtained with the Large Binocular Cameras (LBCs) of the Large Binocular Telescope (LBT). Details on the survey and results from the study of Andromeda XIX (And XIX), Andromeda XXI (And XXI), and Andromeda XXV (And XXV) were presented in Cusano et al. (2013, Paper I), Cusano et al. (2015, Paper II), and Cusano et al. (2016, Paper III), respectively. In this paper we report results on the dwarf spheroidal galaxy (dSph) Andromeda XXVII (And XXVII), which was discovered by Richardson et al. (2011) in the context of the PAndAS survey. The galaxy is located near a portion of Andromeda's northwest stream (NW; see Figure 1 of Richardson et al. 2011). These authors claimed that And XXVII is in the process of being

\footnotetext{
* Based on data collected with the Large Binocular Cameras at the Large Binocular Telescope, PI: G. Clementini.
}

tidally disrupted by M31 and derived only a lower limit of $\geqslant 757 \pm 45 \mathrm{kpc}$ for its heliocentric distance due to the difficulty in measuring the magnitude of the galaxy horizontal branch (HB). Ibata et al. (2013) later found that And XXVII is a member of the thin plane of satellites identified in M31 (Great Plane of Andromeda, GPoA, following the definition of Pawlowski et al. 2013). The heliocentric distance of And XXVII was revised using the tip of the red giant branch (RGB) stars by Conn et al. (2012), who estimated a value of $1255_{-474}^{+42} \mathrm{kpc}$, which would place the galaxy outside the M31 complex. The RGB tip of And XXVII is very scarcely populated, likely due to the disruptive nature of the galaxy. This may have significantly affected Conn et al.'s (2012) determination of distance. Collins et al. (2013) measured a metallicity for And XXVII of $[\mathrm{Fe} / \mathrm{H}]=-2.1 \pm 0.5 \mathrm{dex}$ estimated from the calcium triplet (Ca II) in 11 probable member stars. The radial velocity (RV) of And XXVII measured by the same authors is: $v_{r}=-539.6 \mathrm{~km} \mathrm{~s}^{-1}$, with $\sigma=14.8 \mathrm{~km} \mathrm{~s}^{-1}$. Collins et al. (2013) warned that given the disruptive nature of the object, their estimate is very uncertain. They also identified a significant kinematic substructure around $\sim-500 \mathrm{~km} \mathrm{~s}^{-1}$. Very recently, Martin et al. (2016), from a reanalysis of the PAndAS data, stated that And XXVII is likely a system that is in the final phase of tidal disruption. The widefield LBT observations of And XXVII presented in this paper lend further support to this evidence from the analysis of both the color-magnitude diagram (CMD) and spatial distribution of the variable stars. 
Table 1

Log of And XXVII Observations

\begin{tabular}{lcrcc}
\hline \hline Dates & Filter & $N$ & $\begin{array}{c}\text { Exposure Time } \\
(\mathrm{s})\end{array}$ & $\begin{array}{c}\text { Seeing (FWHM) } \\
(\operatorname{arcsec})\end{array}$ \\
\hline 2011 Oct 20-24 & $B$ & 87 & 400 & $0.8-1$ \\
2011 Nov 27-28 & $B$ & 5 & 400 & $0.8-1$ \\
2011 Oct 20-24 & $V$ & 86 & 400 & $0.8-1$ \\
2011 Nov 27-28 & $V$ & 4 & 400 & $0.8-1$ \\
\hline
\end{tabular}

The paper is organized as follows. Section 2 describes the data collection and processing. Section 3 describes the identification of the variable stars and their characterization. The galaxy CMD is presented in Section 4. Distance and galaxy structure are discussed in Sections 5 and 6. Conclusions are provided in Section 7.

\section{Observations and Data Reduction}

Time-series observations in the $B$ - and $V$-bands of the field around the center coordinates of And XXVII (R.A. $=00^{\mathrm{h}} 37^{\mathrm{m}} 27^{\mathrm{s}}$, decl. $=+45^{\circ} 23^{\prime} 13^{\prime \prime}$; J2000, Richardson et al. 2011) were carried out in 2011 October-November (see Table 1 for the complete log) at the LBT equipped with the LBC. The total LBC's field of view $(\mathrm{FoV})$ covers an area of $\sim 23^{\prime} \times 23^{\prime}$. Observations were obtained under good sky and seeing conditions (see Table 1). The blue camera (LBC-B) was used to acquire the $B$ images, while $V$ imaging was obtained with the red camera (LBC-R). The bias, flat-fielding, and distortion corrections of the raw frames were performed with a dedicated pipeline developed at INAF-OAR. ${ }^{6}$ We performed PSF photometry for the pre-processed images using the DAOPHOT-ALLSTAR-ALLFRAME packages (Stetson 1987, 1994) as described in Paper I. Photometric calibration was performed using the Landolt standard fields L92 and SA113, observed during the same observing run. The calibration equations ${ }^{7}$ are consistent with those derived in Paper I, once differences in airmass and exposure times are accounted for.

\section{Variable Stars}

Variable stars were identified in our photometric catalogs using the same procedure as described in Paper I. We used the variability index (VI) computed in DAOMASTER (Stetson 1994) to search for variable sources. We selected as candidate variables stars with a VI $>1.4$, based on our previous experience with other M31 satellites (And XIX, And XXI, And XXV) and LBT photometry. The average value for non-variable stellar objects placed on the HB and satisfying the quality parameters, $\chi \leqslant 1.5$, $-0.35<$ Sharpness $<0.35$ (see Section 4), is $\langle\mathrm{VI}\rangle=1.03$, $\sigma=0.12$. The value of VI used to select candidate variable stars is thus $3 \sigma$ above the average value for $\mathrm{HB}$ non-variable stars. The selection of variable stars was performed on the photometric catalog for objects satisfying the quality parameters $(\chi$ and Sharpness) mentioned above. The total number of candidate variables that passed our selection criteria is 754 . The $B$ and $V$ light curves of the candidate variables were then analyzed using the Graphical Analyzer of Time Series package (GRaTIS), custom software developed at the Bologna Observatory by P. Montegriffo (see, e.g., Clementini et al. 2000). From this analysis, 90 sources

\footnotetext{
6 http://lbc.oa-roma.inaf.it/commissioning/index.html

$7 \quad B-b=27.696-0.113 \times(b-v) \mathrm{rms}=0.03, V-v=27.542-0.060 \times$ $(b-v) \mathrm{rms}=0.03$.
}

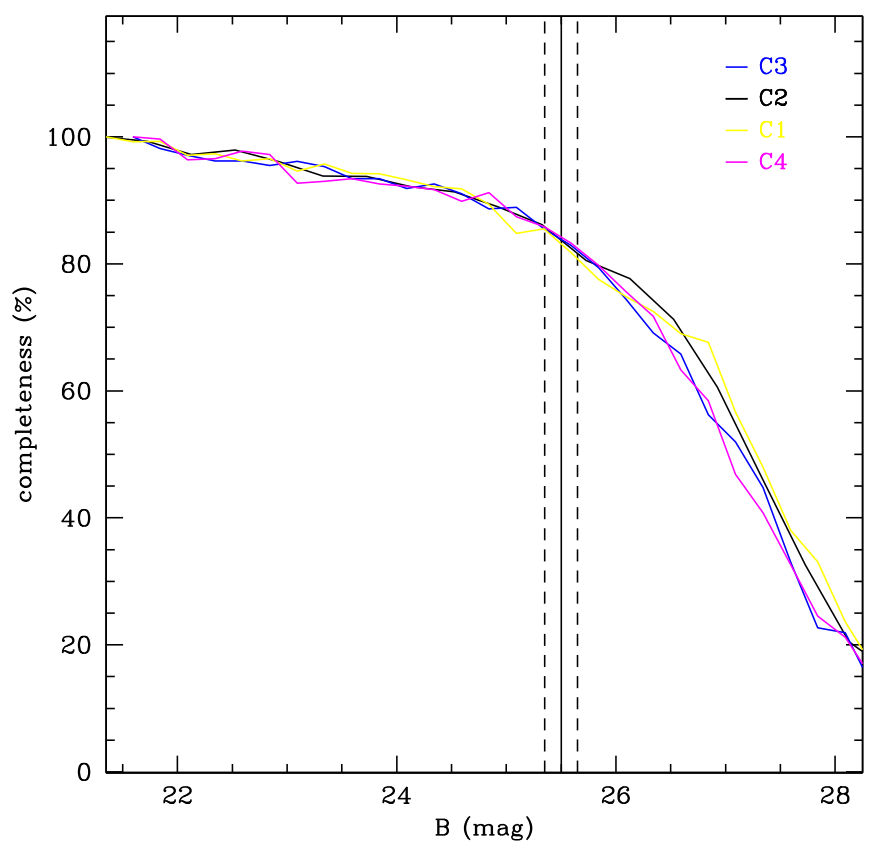

Figure 1. Completeness of the photometric catalogs in the four CCDs (hereinafter $\mathrm{C} 1, \mathrm{C} 2, \mathrm{C} 3$, and C4) of the $\mathrm{LBC}$ mosaic. The blue line is $\mathrm{C} 3$, the yellow line is $\mathrm{C} 1$, the magenta line is $\mathrm{C} 4$, and the black line is $\mathrm{C} 2$. The vertical solid line marks the average $B$ magnitude of the RR Lyrae stars, and the dashed lines are the $1 \sigma$ boundaries.

were confirmed to vary. Of them, 89 are classified as RR Lyrae stars and 1 as an anomalous Cepheid (AC), based on the pulsation properties and the position in the CMD. The lowest value of VI for bona fide RR Lyrae stars identified in this work is VI $=1.52$, hence the VI limit we chose to select candidate variable stars is loose enough to ensure we did not miss candidate variables. Therefore, the completeness of our RR Lyrae stars catalog is mostly driven by the photometric completeness at the HB level. This was estimated using artificial star tests and at the level of $\mathrm{HB}$ is $\sim 83 \%$, as shown in Figure 1. The figure shows the trend of the completeness in the $B$-band magnitude for each of the four CCDs of the LBC mosaic, and clearly there is no significant difference among the four CCDs, especially at the average magnitude of the RR Lyrae stars (solid vertical line). Finally, the incompleteness of our RR Lyrae stars catalog is $\sim 17 \%$, hence we are probably missing 10 to 20 RR Lyrae stars in the And XXVII field.

The coordinates and properties of the confirmed variable stars are listed in Table 2, where column 1 gives the star identifier; columns 2 and 3 are, respectively, the right ascension and declination (J2000.0 epoch) obtained from our astrometrized catalogs; column 4 provides the type of variability, with a question mark identifying stars whose classifications are uncertain; columns 5 and 6 give, respectively, the period and the Heliocentric Julian Day (HJD) of maximum light; columns 7 and 9 are the intensity-averaged mean $B$ and $V$ magnitudes; columns 8 and 10 list the corresponding amplitudes of light variation. Examples of light curves, in both filters, are shown in Figure 2. Observations were performed on five consecutive nights in October and two consecutive nights in November. The data are mostly biased by the one-day alias due to the alternation of night-to-day observations and are thus marginally affecting the period determination of the RR Lyrae stars that in all cases is $<1$ day. The full catalog of light curves is available in the electronic version of the paper. 
Table 2

Identification and Properties of the Variable Stars Detected in And XXVII

\begin{tabular}{|c|c|c|c|c|c|c|c|c|c|}
\hline Name & $\begin{array}{c}\alpha \\
(2000)\end{array}$ & $\begin{array}{c}\delta \\
(2000)\end{array}$ & Type & $\begin{array}{c}P \\
\text { (days) }\end{array}$ & $\begin{array}{c}\text { Epoch }(\max ) \\
\text { HJD }(-2455000)\end{array}$ & $\begin{array}{c}\langle B\rangle \\
(\mathrm{mag})\end{array}$ & $\begin{array}{c}A_{B} \\
(\mathrm{mag})\end{array}$ & $\begin{array}{l}\langle V\rangle \\
(\mathrm{mag})\end{array}$ & $\begin{array}{c}A_{V} \\
(\mathrm{mag})\end{array}$ \\
\hline V1 & $00: 37: 31.893$ & $+45: 23: 54.12$ & $\mathrm{RRab}$ & 0.6171 & 53.792 & 25.38 & 0.62 & 24.94 & 0.49 \\
\hline $\mathrm{V} 2$ & $00: 37: 32.950$ & $+45: 22: 58.27$ & $\mathrm{AC}$ & 1.2502 & 53.085 & 23.79 & 1.73 & 23.48 & 1.43 \\
\hline V3 & $00: 37: 33.655$ & $+45: 24: 40.61$ & $\mathrm{RRc}$ & 0.3091 & 53.633 & 25.19 & 0.74 & 24.91 & 0.53 \\
\hline V4 & $00: 37: 19.667$ & $+45: 24: 27.33$ & $\mathrm{RRc}$ & 0.3857 & 53.272 & 25.38 & 0.82 & 25.08 & 0.59 \\
\hline V5 & $00: 37: 36.643$ & $+45: 22: 58.03$ & $\mathrm{RRab}$ & 0.6048 & 54.080 & 25.72 & 1.05 & 25.37 & 0.82 \\
\hline V6 & $00: 37: 19.271$ & $+45: 21: 17.88$ & $\mathrm{RRc}$ & 0.3642 & 53.600 & 25.61 & 0.59 & 25.32 & 0.46 \\
\hline V7 & $00: 37: 28.721$ & $+45: 26: 12.74$ & RRab & 0.5798 & 53.762 & 25.78 & 1.04 & 25.25 & 0.55 \\
\hline V8 & $00: 37: 25.769$ & $+45: 19: 50.86$ & $\mathrm{RRab}$ & 0.5999 & 53.421 & 25.55 & 0.94 & 25.14 & 0.62 \\
\hline V9 & $00: 37: 22.045$ & $+45: 26: 39.96$ & $\mathrm{RRab}$ & 0.6910 & 53.292 & 25.56 & 0.66 & 25.19 & 0.66 \\
\hline V10 & $00: 37: 23.724$ & $+45: 19: 30.90$ & RRab & 0.6226 & 53.817 & 25.74 & 0.52 & 25.33 & 0.37 \\
\hline V11 & $00: 37: 16.889$ & $+45: 26: 08.05$ & $\mathrm{RRc}$ & 0.3552 & 53.428 & 25.41 & 0.69 & 25.06 & 0.42 \\
\hline V12 & $00: 37: 19.295$ & $+45: 19: 31.97$ & $\mathrm{RRc}$ & 0.2432 & 53.856 & 25.30 & 0.69 & 25.05 & 0.57 \\
\hline V13 & $00: 37: 37.243$ & $+45: 27: 03.68$ & $\mathrm{RRc}$ & 0.3635 & 53.735 & 25.38 & 0.72 & 24.99 & 0.53 \\
\hline V14 & $00: 37: 38.799$ & $+45: 26: 48.76$ & $\mathrm{RRab}$ & 0.6710 & 53.271 & 25.22 & 0.94 & 24.76 & 0.70 \\
\hline V15 & $00: 37: 44.006$ & $+45: 21: 10.15$ & $\mathrm{RRab}$ & 0.5645 & 53.783 & 25.78 & 0.97 & 25.53 & 0.85 \\
\hline V16 & $00: 37: 21.577$ & $+45: 18: 40.74$ & RRab & 0.5881 & 53.459 & 25.72 & 1.16 & 25.35 & 0.78 \\
\hline V17 & $00: 37: 46.582$ & $+45: 23: 51.84$ & $\mathrm{RRab}$ & 0.6586 & 53.390 & 25.64 & 0.67 & 25.30 & 0.59 \\
\hline V18 & $00: 37: 29.435$ & $+45: 28: 15.86$ & $\mathrm{RRab}$ & 0.5793 & 53.425 & 25.57 & 1.09 & 25.21 & 0.89 \\
\hline V19 & $00: 37: 30.249$ & $+45: 18: 03.82$ & RRab & 0.6181 & 53.987 & 25.33 & 1.30 & 25.05 & 1.18 \\
\hline V20 & $00: 37: 07.677$ & $+45: 26: 04.87$ & $\mathrm{RRab}$ & 0.5612 & 53.679 & 25.30 & 1.49 & 24.92 & 0.99 \\
\hline V21 & $00: 37: 40.518$ & $+45: 27: 58.79$ & $\mathrm{RRc}$ & 0.4357 & 53.691 & 25.42 & 0.46 & 24.99 & 0.31 \\
\hline V22 & $00: 37: 12.966$ & $+45: 18: 22.55$ & $\mathrm{RRc}$ & 0.2925 & 53.985 & 25.33 & 0.67 & 25.12 & 0.57 \\
\hline V23 & $00: 37: 38.962$ & $+45: 28: 28.95$ & $\mathrm{RRab}$ & 0.5570 & 53.857 & 25.11 & 1.14 & 24.70 & 0.96 \\
\hline V24 & $00: 37: 06.459$ & $+45: 19: 45.37$ & $\mathrm{RRab}$ & 0.5746 & 53.559 & 25.41 & 1.19 & 25.10 & 0.96 \\
\hline V25 & $00: 37: 45.060$ & $+45: 18: 43.87$ & $\mathrm{RRab}$ & 0.7150 & 53.391 & 25.52 & 0.71 & 25.11 & 0.70 \\
\hline V26 & $00: 37: 01.535$ & $+45: 24: 21.95$ & $\mathrm{RRab}$ & 0.5535 & 53.588 & 25.66 & 1.00 & 25.26 & 1.03 \\
\hline V27 & $00: 37: 45.003$ & $+45: 28: 36.34$ & $\mathrm{RRc}$ & 0.3938 & 53.147 & 25.52 & 0.56 & 25.10 & 0.35 \\
\hline V28 & $00: 37: 52.395$ & $+45: 19: 57.61$ & RRab & 0.5870 & 53.910 & 25.58 & 0.95 & 25.13 & 0.87 \\
\hline V29 & $00: 37: 17.317$ & $+45: 30: 03.48$ & $\mathrm{RRc}$ & 0.3601 & 54.076 & 25.34 & 0.44 & 24.88 & 0.38 \\
\hline V30 & 00:36:59.737 & $+45: 25: 39.13$ & RRab & 0.5541 & 53.887 & 25.50 & 1.04 & 25.14 & 0.69 \\
\hline V31 & 00:37:56.368 & $+45: 25: 06.03$ & RRab & 0.5408 & 53.803 & 25.56 & 1.05 & 25.25 & 0.98 \\
\hline V32 & $00: 37: 57.393$ & $+45: 21: 23.21$ & $\mathrm{RRc}$ ? & 0.2793 & 53.931 & 25.44 & 0.47 & 25.27 & 0.47 \\
\hline V33 & $00: 37: 56.332$ & $+45: 20: 25.79$ & $\mathrm{RRab}$ & 0.5492 & 53.824 & 25.50 & 0.82 & 25.23 & 0.50 \\
\hline V34 & $00: 37: 00.936$ & $+45: 18: 26.79$ & RRab & 0.5579 & 53.875 & 25.54 & 1.46 & 25.18 & 1.00 \\
\hline V35 & $00: 37: 58.868$ & $+45: 20: 16.64$ & $\mathrm{RRc}$ & 0.3579 & 53.544 & 25.67 & 0.58 & 25.30 & 0.43 \\
\hline V36 & 00:38:00.070 & $+45: 21: 13.33$ & $\mathrm{RRc}$ & 0.3641 & 53.781 & 25.60 & 0.66 & 25.21 & 0.67 \\
\hline V37 & 00:38:00.481 & $+45: 24: 46.02$ & $\mathrm{RRab}$ & 0.5996 & 53.525 & 25.35 & 1.31 & 24.99 & 0.96 \\
\hline V38 & $00: 37: 52.645$ & $+45: 17: 32.73$ & $\mathrm{RRc}$ & 0.3987 & 53.928 & 25.27 & 0.63 & 24.92 & 0.45 \\
\hline V39 & $00: 36: 55.883$ & $+45: 27: 09.36$ & $\mathrm{RRc}$ & 0.3534 & 53.504 & 25.51 & 0.82 & 25.14 & 0.56 \\
\hline V40 & $00: 38: 02.723$ & $+45: 24: 46.06$ & $\mathrm{RRc}$ & 0.3395 & 53.616 & 25.50 & 0.72 & 25.28 & 0.59 \\
\hline V41 & $00: 37: 13.142$ & $+45: 14: 50.52$ & RRab & 0.5888 & 53.486 & 25.50 & 1.05 & 25.20 & 1.02 \\
\hline V42 & 00:36:51.394 & $+45: 25: 50.83$ & $\mathrm{RRc}$ & 0.3550 & 53.660 & 25.55 & 0.57 & 25.27 & 0.56 \\
\hline V43 & $00: 37: 56.003$ & $+45: 29: 09.95$ & RRab & 0.6087 & 53.686 & 25.66 & 0.97 & 25.24 & 0.72 \\
\hline V44 & $00: 37: 25.859$ & $+45: 32: 50.55$ & RRab? & 0.6312 & 53.322 & 25.37 & 0.45 & 25.12 & 0.53 \\
\hline V45 & $00: 36: 49.108$ & $+45: 20: 47.83$ & $\mathrm{RRab}$ & 0.5315 & 53.758 & 25.37 & 1.28 & 25.03 & 1.18 \\
\hline V46 & $00: 37: 24.064$ & $+45: 13: 16.69$ & RRab & 0.5855 & 54.116 & 25.78 & 0.82 & 25.46 & 0.90 \\
\hline V47 & 00:38:02.920 & $+45: 18: 20.73$ & RRab & 0.5293 & 53.342 & 25.67 & 1.14 & 25.29 & 0.70 \\
\hline V48 & $00: 38: 06.624$ & $+45: 20: 36.47$ & $\mathrm{RRc}$ & 0.3501 & 53.816 & 25.58 & 0.54 & 25.26 & 0.45 \\
\hline V49 & $00: 38: 05.333$ & $+45: 19: 20.00$ & $\mathrm{RRc}$ & 0.3541 & 53.015 & 25.54 & 0.50 & 25.21 & 0.31 \\
\hline V50 & $00: 36: 48.952$ & $+45: 19: 02.07$ & $\mathrm{RRab}$ & 0.6451 & 53.359 & 25.56 & 1.17 & 25.14 & 0.76 \\
\hline V51 & $00: 37: 13.308$ & $+45: 13: 20.85$ & $\mathrm{RRc}$ & 0.3558 & 53.410 & 25.53 & 0.61 & 25.28 & 0.51 \\
\hline V52 & $00: 36: 47.422$ & $+45: 27: 31.80$ & RRab & 0.5627 & 53.761 & 25.20 & 1.21 & 24.84 & 0.85 \\
\hline V53 & $00: 36: 52.857$ & $+45: 30: 18.98$ & RRab & 0.5980 & 53.706 & 25.42 & 0.78 & 25.06 & 0.69 \\
\hline V54 & $00: 38: 11.223$ & $+45: 21: 26.58$ & RRab & 0.5754 & 54.079 & 25.41 & 1.10 & 25.12 & 1.02 \\
\hline V55 & 00:37:09.934 & $+45: 12: 53.19$ & RRab & 0.6226 & 53.920 & 25.53 & 0.79 & 25.24 & 0.57 \\
\hline V56 & $00: 38: 02.164$ & $+45: 30: 14.28$ & $\mathrm{RRab}$ & 0.5794 & 53.853 & 25.60 & 0.95 & 25.26 & 0.84 \\
\hline V57 & $00: 36: 42.634$ & $+45: 21: 18.38$ & RRab & 0.5357 & 53.638 & 25.49 & 1.18 & 25.29 & 0.96 \\
\hline V58 & 00:38:10.002 & $+45: 18: 59.73$ & $\mathrm{RRab}$ & 0.5395 & 53.369 & 25.62 & 1.00 & 25.25 & 0.79 \\
\hline V59 & $00: 37: 43.102$ & $+45: 34: 14.31$ & $\mathrm{RRab}$ & 0.6000 & 53.707 & 25.42 & 1.19 & 25.04 & 0.92 \\
\hline V60 & $00: 36: 39.192$ & $+45: 23: 25.77$ & $\mathrm{RRab}$ & 0.5788 & 53.866 & 25.47 & 0.94 & 25.09 & 0.94 \\
\hline V61 & $00: 38: 16.025$ & $+45: 24: 01.56$ & RRc? & 0.3830 & 53.841 & 25.43 & 0.75 & 25.20 & 0.76 \\
\hline V62 & $00: 36: 37.523$ & $+45: 23: 46.74$ & $\mathrm{RRc}$ & 0.4281 & 53.797 & 25.41 & 0.58 & 24.95 & 0.22 \\
\hline V63 & $00: 38: 15.170$ & $+45: 19: 44.42$ & RRab & 0.6023 & 53.829 & 25.10 & 0.87 & 24.73 & 0.71 \\
\hline
\end{tabular}


Table 2

(Continued)

\begin{tabular}{|c|c|c|c|c|c|c|c|c|c|}
\hline Name & $\begin{array}{c}\alpha \\
(2000)\end{array}$ & $\begin{array}{c}\delta \\
(2000)\end{array}$ & Type & $\begin{array}{c}P \\
\text { (days) }\end{array}$ & $\begin{array}{c}\text { Epoch }(\max ) \\
\text { HJD }(-2455000)\end{array}$ & $\begin{array}{c}\langle B\rangle \\
(\mathrm{mag})\end{array}$ & $\begin{array}{c}A_{B} \\
(\mathrm{mag})\end{array}$ & $\begin{array}{c}\langle V\rangle \\
(\mathrm{mag})\end{array}$ & $\begin{array}{c}A_{V} \\
(\mathrm{mag})\end{array}$ \\
\hline V64 & $00: 36: 42.559$ & $+45: 17: 17.99$ & RRab & 0.5537 & 53.877 & 25.41 & 1.19 & 25.10 & 0.96 \\
\hline V65 & 00:38:07.107 & $+45: 31: 17.21$ & RRab & 0.6035 & 53.411 & 25.57 & 0.87 & 25.16 & 0.53 \\
\hline V66 & $00: 38: 17.987$ & $+45: 21: 20.02$ & RRab & 0.7383 & 53.309 & 25.34 & 0.52 & 24.77 & 0.35 \\
\hline V67 & $00: 38: 14.697$ & $+45: 17: 42.86$ & RRab & 0.5621 & 53.544 & 25.76 & 0.90 & 25.45 & 0.68 \\
\hline V68 & $00: 36: 45.329$ & $+45: 14: 56.17$ & $\mathrm{RRc}$ & 0.3682 & 53.304 & 25.49 & 0.67 & 25.21 & 0.50 \\
\hline V69 & $00: 38: 13.766$ & $+45: 16: 28.40$ & RRab & 0.5715 & 53.933 & 25.70 & 0.90 & 25.33 & 0.98 \\
\hline V70 & $00: 36: 33.155$ & $+45: 24: 24.17$ & $\mathrm{RRc}$ & 0.3547 & 53.748 & 25.54 & 0.58 & 25.15 & 0.37 \\
\hline V71 & 00:38:20.719 & $+45: 20: 47.63$ & $\mathrm{RRc}$ & 0.3594 & 53.929 & 25.45 & 0.66 & 25.15 & 0.58 \\
\hline V72 & $00: 38: 18.355$ & $+45: 27: 54.69$ & RRab & 0.6236 & 53.679 & 25.64 & 0.84 & 25.25 & 0.71 \\
\hline V73 & $00: 38: 06.155$ & $+45: 32: 45.88$ & RRab & 0.6524 & 53.969 & 25.57 & 1.22 & 25.27 & 1.16 \\
\hline V74 & $00: 38: 12.060$ & $+45: 15: 24.61$ & $\mathrm{RRab}$ & 0.5616 & 53.699 & 25.56 & 0.96 & 25.12 & 0.56 \\
\hline V75 & 00:38:05.872 & $+45: 13: 29.96$ & $\mathrm{RRc}$ & 0.3487 & 53.877 & 25.50 & 0.64 & 25.20 & 0.56 \\
\hline V76 & $00: 36: 35.681$ & $+45: 28: 17.18$ & $\mathrm{RRc}$ & 0.3642 & 53.833 & 25.53 & 0.65 & 25.20 & 0.48 \\
\hline V77 & 00:38:23.292 & $+45: 22: 27.00$ & RRab & 0.5306 & 53.267 & 25.65 & 1.15 & 25.30 & 0.96 \\
\hline V78 & $00: 38: 14.075$ & $+45: 15: 18.82$ & $\mathrm{RRc}$ & 0.3333 & 53.837 & 25.50 & 0.68 & 25.24 & 0.65 \\
\hline V79 & $00: 36: 29.952$ & $+45: 23: 43.59$ & RRc? & 0.2924 & 53.494 & 25.37 & 0.70 & 25.19 & 0.48 \\
\hline V80 & $00: 38: 23.752$ & $+45: 20: 48.11$ & $\mathrm{RRc}$ & 0.3648 & 53.352 & 25.61 & 0.74 & 25.31 & 0.64 \\
\hline V81 & $00: 36: 30.046$ & $+45: 25: 46.60$ & RRab & 0.5595 & 53.968 & 25.26 & 1.01 & 24.89 & 0.82 \\
\hline V82 & 00:38:09.009 & $+45: 12: 59.08$ & $\mathrm{RRc}$ & 0.2547 & 53.786 & 25.49 & 0.69 & 25.24 & 0.38 \\
\hline V83 & $00: 38: 27.464$ & $+45: 23: 03.51$ & $\mathrm{RRab}$ & 0.5628 & 53.780 & 25.50 & 1.15 & 25.16 & 0.92 \\
\hline V84 & $00: 36: 31.312$ & $+45: 28: 59.64$ & RRab & 0.6628 & 53.385 & 25.33 & 1.00 & 24.83 & 0.71 \\
\hline V85 & $00: 38: 20.435$ & $+45: 15: 59.67$ & RRab & 0.5575 & 53.929 & 25.87 & 1.38 & 25.48 & 1.11 \\
\hline V86 & $00: 38: 28.400$ & $+45: 20: 51.17$ & $\mathrm{RRab}$ & 0.5453 & 54.120 & 25.73 & 1.10 & 25.29 & 0.99 \\
\hline V87 & $00: 36: 28.323$ & $+45: 15: 54.01$ & RRab & 0.7117 & 53.641 & 25.45 & 0.77 & 25.10 & 0.56 \\
\hline V88 & $00: 38: 32.760$ & $+45: 26: 30.92$ & RRab & 0.6063 & 53.786 & 25.47 & 0.73 & 25.07 & 0.56 \\
\hline V89 & $00: 36: 19.903$ & $+45: 21: 49.53$ & RRab & 0.5905 & 53.809 & 25.29 & 1.68 & 25.01 & 1.47 \\
\hline V90 & $00: 36: 21.505$ & $+45: 18: 27.19$ & RRab & 0.5959 & 53.657 & 25.41 & 1.03 & 25.15 & 0.64 \\
\hline
\end{tabular}

\subsection{RR Lyrae Stars}

In the field of And XXVII we have discovered a total of 89 RR Lyrae stars, of which 58 are fundamental mode (RRab) and 31 are first-overtone (RRc) pulsators, according to the periods and amplitudes of light variation. RR Lyrae stars are excellent tracers of an old stellar population (e.g., Clementini 2010; Marconi et al. 2015, and references therein). Their presence in And XXVII shows that this galaxy started forming stars $\geqslant 10$ Gyr ago. The spatial distribution of the variable stars discovered in the FoV of And XXVII is shown in Figure 3. The highest concentration of RR Lyrae stars occurs in CCD3 (labeled C3 in the figure), where there are $32 \mathrm{RR}$ Lyrae to compare with 28 in $\mathrm{C} 2,21$ in $\mathrm{C} 1$, and 7 in $\mathrm{C} 4$. Furthermore, the very sparse spatial distributions of these variable stars suggest that And XXVII is in the phase of tidal disruption, as the RR Lyrae stars are spread all over the LBC FoV. However, given the proximity to M31 we cannot rule out that some of these RR Lyrae stars might belong to Andromeda's halo. To estimate how many M31 halo RR Lyrae stars we can expect in the LBC FoV centered on And XXVII, we considered a number of different arguments. First, we assumed the surface density profile from Ibata et al. (2013). Despite the complexity of the M31 halo, Ibata et al. (2013) found that the azimuthally averaged projected star-count profile for stars in the metallicity range $-2.5<[\mathrm{Fe} / \mathrm{H}]<-1.7$ does not have particular features and possesses a power law of $\Gamma=-2.3 \pm 0.02$. Then, we considered that Jeffery et al. (2011) found five and zero RR Lyrae stars in two HST/ACS fields at a distance of $35 \mathrm{kpc}$ from the M31 center. If we apply Ibata et al.'s power law to the sample of RR Lyrae stars found by Jeffery et al. (2011) after rescaling for the different area surveyed by our LBC images $\left(\sim 0.15 \mathrm{deg}^{2}\right)$ and the HST/ACS $\left(\sim 0.008 \mathrm{deg}^{2}\right)$ and the different distance to the center of M31 (the projected distance to And XXVII is $60 \mathrm{kpc}$ ), we find a possible contamination of $\sim 14$ RR Lyrae stars from the M31 halo in And XXVII's LBC field. However, we note that the number of contaminant RR Lyrae stars could be larger than this estimate, as And XXVII lies in a region of the M31 halo where stars in the metallicity range $-2.5<[\mathrm{Fe} / \mathrm{H}]<-1.7$ form streams and are all around And XXVII, as shown in Figure 9 of Ibata et al. (2014). Thus, decontamination of And XXVII stars from field objects is a difficult task.

The period distribution of the RR Lyrae stars identified in the field of And XXVII is shown by the histogram in Figure 4. The average period of the 58 RRab stars is $\left\langle P_{\mathrm{ab}}\right\rangle=0.59$ days ( $\sigma=0.05$ day), while that for the $31 \mathrm{RRc}$ is $\left\langle P_{\mathrm{c}}\right\rangle=0.35$ days ( $\sigma=0.04$ day). Considering that $\sim 14$ RR Lyrae stars could belong to the M31 halo, we performed a bootstrap re-sampling of the data, removing a total of 14 RR Lyrae stars. Among these we randomly removed $9 \mathrm{RRab}$ and $5 \mathrm{RRc}$, consistent with the fraction of RRc over total RR Lyrae stars found. Both the average period and related $\sigma$ of RRab and RRc stars, separately, do not change significantly, giving us confidence that the RR Lyrae stars of And XXVII have the same properties of those belonging to the surrounding M31 halo.

Based on the average period of the fundamental mode pulsators, And XXVII would be classified as an Oosterhoff-Intermediate (Oo Int)/OoI system (Oosterhoff 1939; Catelan 2009); however, we note that And XXVII has the lowest value of $\left\langle P_{\mathrm{ab}}\right\rangle$ among the four M31 satellites we have investigated so far based on LBT data. The fraction of RRc to the total number of RR Lyrae stars is 

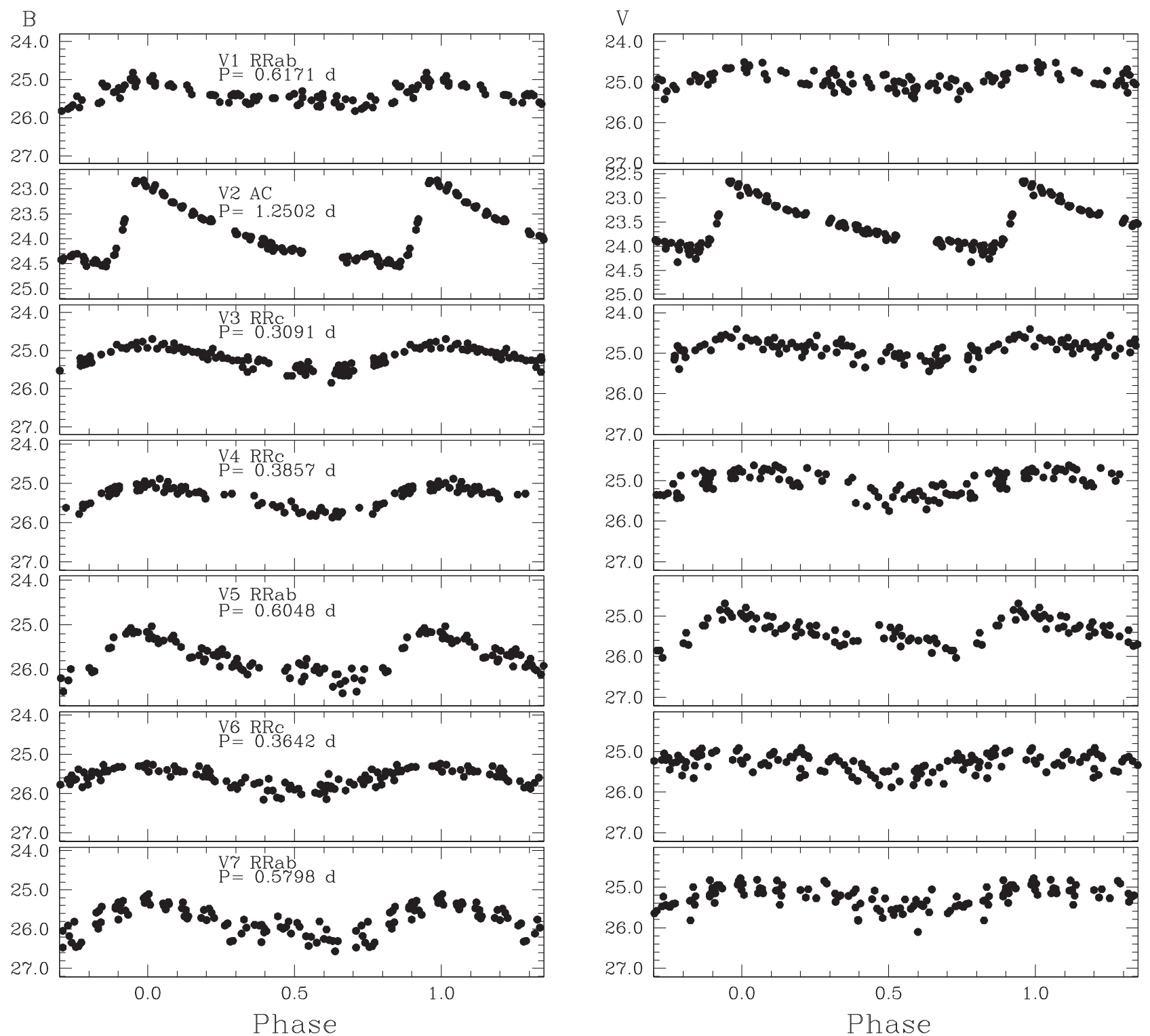

Figure 2. $B$ - (left panels) and $V$-band (right panels) light curves for different types of variable stars identified in And XXVII. The typical internal errors of the singleepoch data range from 0.01 at $B \sim 22.8 \mathrm{mag}$ (corresponding to the maximum light of the AC) to $0.30 \mathrm{mag}$ at $B \sim 26.8 \mathrm{mag}$ (corresponding to the minimum light of a fundamental mode RR Lyrae), and similarly, from $0.01 \mathrm{mag}$ at $V \sim 22.6 \mathrm{mag}$ to $0.30 \mathrm{mag}$ at $V \sim 26.8 \mathrm{mag}$. (An extended version of this figure is available.)

$f_{c}=N_{c} / N_{\mathrm{ab}+\mathrm{c}}=0.35 \pm 0.07$. This is much closer to the value expected for Oosterhoff II (Oo II; $f_{c} \sim 0.44$ ) than Oosterhoff I (Oo I; $\left.f_{c} \sim 0.17\right)$ systems (Catelan 2009). Even in the extreme case that $5 \mathrm{RRc}$ variables belong to the M31 halo and all the RRab stars belong to And XXVII, the fraction would become $f_{c}=0.30$, which is still rather high for an Oo I system. Hence, while the average period of the RRab stars suggests a classification as an Oo-Int/Oo I type, the RRc fraction would suggest a classification more similar to the Oo II type. The left panel of Figure 5 shows the period-amplitude diagram (also known as a Bailey diagram, Bailey 1902) of the RR Lyrae stars in And XXVII. The solid lines are the loci defined by RR Lyrae stars in the Oo I Galactic globular cluster M3 (lower line) and the Oo II globular cluster $\omega$ Cen (upper line), according to Clement \& Rowe (2000). The majority of the RR Lyrae stars in And XXVII are placed near the locus of the Oo I systems and there are no differences among RR Lyrae stars located in different parts of the LBC FoV (see
Sections 4 and 5 and and Table 3). We conclude that the $\left\langle P_{\mathrm{ab}}\right\rangle$ value and the Bailey diagram suggest a OoI/Int system classification for And XXVII.

\subsection{Metallicity}

The metallicities for the RRab stars in And XXVII were derived using the relation of Alcock et al. (2000; see their Equation (1)). The resulting metallicity distribution is shown in Figure 6. A Gaussian fit of this distribution peaks at $[\mathrm{Fe} / \mathrm{H}]=-1.62 \mathrm{dex}(\sigma=0.23 \mathrm{dex})$. This value is consistent with the photometric estimate of $[\mathrm{Fe} / \mathrm{H}]=-1.7 \pm 0.2 \mathrm{dex}$ obtained in the discovery paper (Richardson et al. 2011) by isochrone-fitting of the galaxy CMD, and is within $1 \sigma$ from the spectroscopic estimate of $[\mathrm{Fe} / \mathrm{H}]=-2.1 \pm 0.5$ dex by Collins et al. (2013). A photometric estimate of the RR Lyrae metallicity can also be derived from the $\phi_{31}$ parameter of the Fourier decomposition of the light curve (see, e.g., Simon \& Teays 1982, 


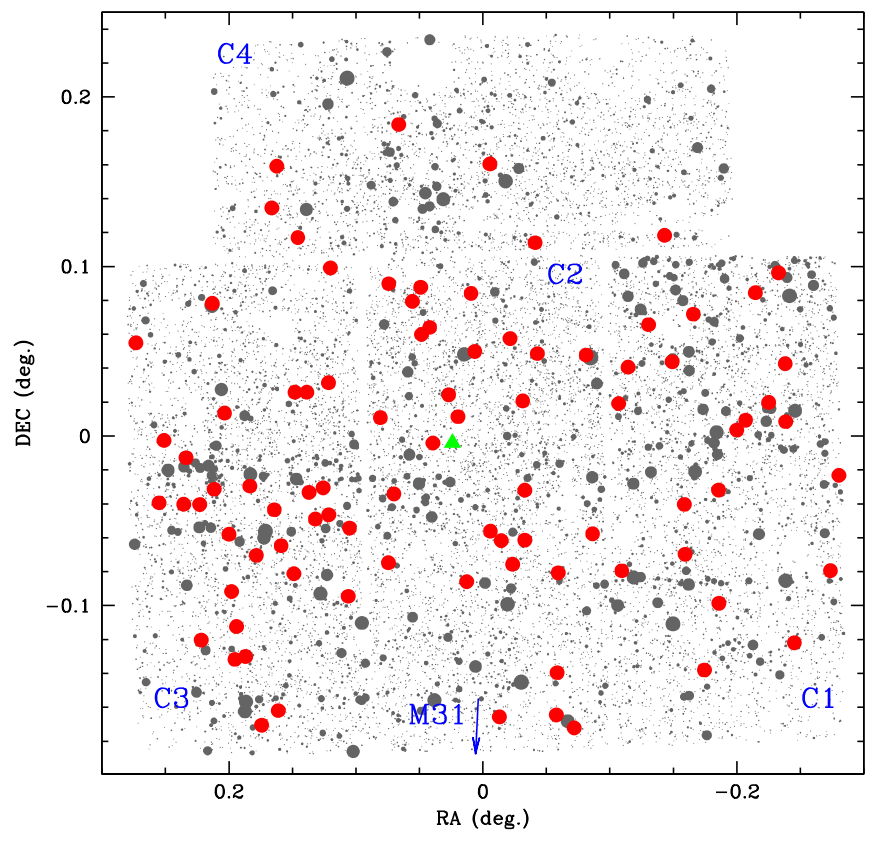

Figure 3. Spatial distribution of the variable stars discovered in the whole LBT FoV. The four CCDs of the LBC mosaic are labeled. The red filled circles mark the RR Lyrae stars, and the green filled triangle is the AC. The gray points are non-variable sources, selected as to have DAOPHOT-quality image parameters: $-0.35 \leqslant$ sharpness $\leqslant 0.35$ and $\chi<1.5$. Their size is inversely proportional to the source $V$-magnitude. Contamination by Milky Way (MW) sources and background galaxies has not been removed (see Section 4). There are 32 RR Lyrae stars displayed in C3, 28 in C2, 21 in C1, and 7 in C4. The blue arrow points to the direction of M31. North is up, and east is to the left.

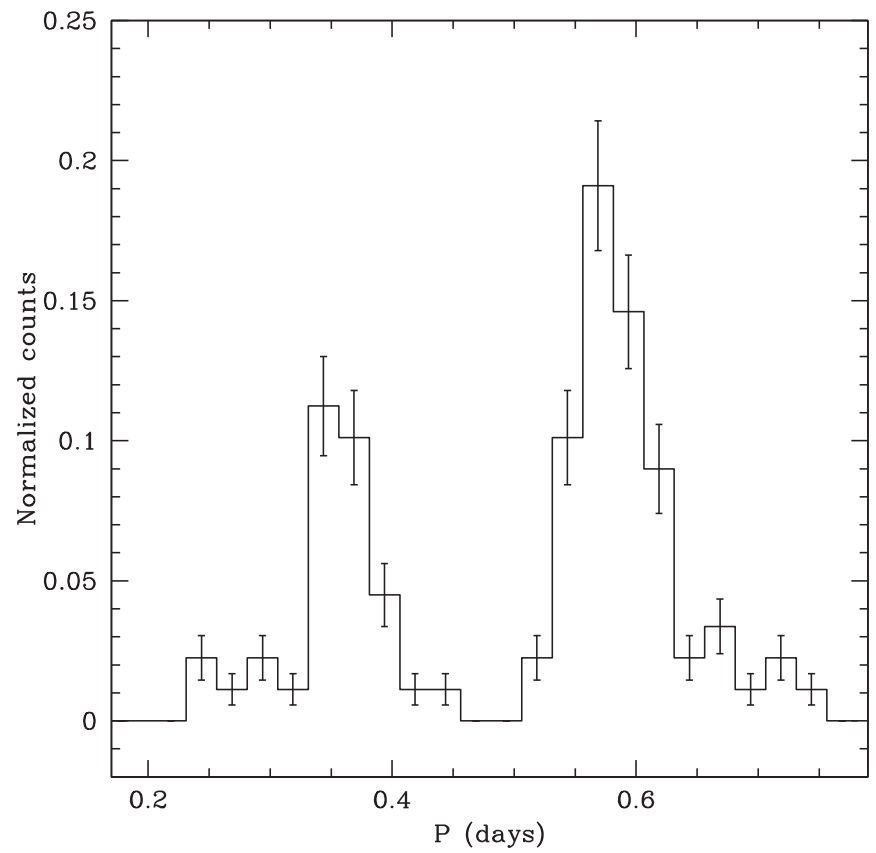

Figure 4. Period distribution of the RR Lyrae stars identified in the field of And XXVII. The bin size is 0.025 days.

Jurcsik \& Kovacs 1996, Cacciari et al. 2005). We performed a sine Fourier decomposition of the $V$-band light curves of the RRab stars in our sample deriving the normalized Fourier parameter $\phi_{31}$ and the deviation parameter $D_{m}$, which measures the regularity of the light curve (Jurcsik \& Kovacs 1996). According to Jurcsik \&
Kovacs (1996) a reliable metallicity can be estimated from the $\phi_{31}$ parameter only if the light curve satisfies the condition $D_{m}<3$.

Among the RRab stars in our sample, only V67 satisfies this condition. ${ }^{8}$ The light curve of V67 is shown in Figure 2. We applied Equation (3) in Jurcsik \& Kovacs (1996) and derived the metallicity $[\mathrm{Fe} / \mathrm{H}]_{J K 96}=-1.75 \pm 0.49$ dex on the Jurcsik \& Kovacs metallicity scale, which becomes $[\mathrm{Fe} / \mathrm{H}]_{\mathrm{C} 09}=-1.86 \pm 0.50 \mathrm{dex}$ once transformed to the Carretta et al. (2009) metallicity scale using Equation (3) from Kapakos et al. (2011). All these values are consistent within their errors with the peak value of the distribution obtained by applying Alcock et al.'s method (see Figure 6).

\subsection{The $A C$}

One of the variable stars identified in And XXVII (V2) falls in the instability strip about one magnitude brighter than the galaxy HB level (see Figure 9). Following the same procedure adopted in Paper I, we have compared V2 with the periodWeseneheit $(P W)$ relations of Large Magellanic Cloud (LMC) ACs from Ripepi et al. (2014) ${ }^{9}$ and the $P W$ relations of LMC classical Cepheids (CCs) from Jacyszyn-Dobrzeniecka et al. 2016). These comparisons are shown in the left and right panels of Figure 7, respectively, which were drawn assuming And XXVII's distance is the distance inferred from the RR Lyrae stars (see Section 5). We have also plotted in the two panels of Figure 7 the ACs we have identified in And XIX, And XXI, and And XXV.

Figure 7 shows that $\mathrm{V} 2$ follows the $P W$ relation for fundamental mode ACs, while it can be definitely ruled out that the star is a short-period $\mathrm{CC}$ (see the right panel of Figure 7). The AC in And XXVII can be interpreted as the result of a merging in a binary system as old as the RR Lyrae stars in which mass-transfer acted in the last 1-2 Gyr. The single young star scenario can be excluded considering that in the CMD there is no evidence of a young stellar component (see Section 4).

As in previous papers of this series, we computed the specific frequency of ACs in And XXVII assuming that V2 is the only $\mathrm{AC}$ in the galaxy, and compared it with results for other galaxies. This is shown in Figure 8, where the ACspecific frequencies of other MW (blue filled circles) and M31 (green and red filled squares) satellites are taken from Paper III. The blue ridge line shows the relation obtained from all the dwarf satellites in the plot. The M31 satellites that are off the GPoA (red squares) seem to follow a different trend shown by the red dashed line. The sample is still statistically too poor to conclude that off-plane and on-plane satellites obey different correlations between ACs' specific frequencies and luminosities or metallicities, but the satellites studied so far seem to suggest that this may be the case.

\section{CMD and Projected Spatial Distributions}

The CMD of the sources in the whole LBC FoV is presented in Figure 9. Only sources with DAOPHOT-quality image parameters: $\quad-0.35 \leqslant$ sharpness $\leqslant 0.35$ and $\chi<1.5$ are

\footnotetext{
8 According to Cacciari et al. (2005) variables satisfying the relaxed condition $D_{m}<5$ could also be used. There are three RR Lyrae stars with $3<D_{m}<5$. They provide very scattered and unreliable metallicity values, hence we did not consider them.

9 Ripepi et al.'s (2014) relations were derived for the $V$ - and $I$-bands, and we have converted them to $B$ and $V$ using Equation (12) of Marconi et al. (2004).
} 

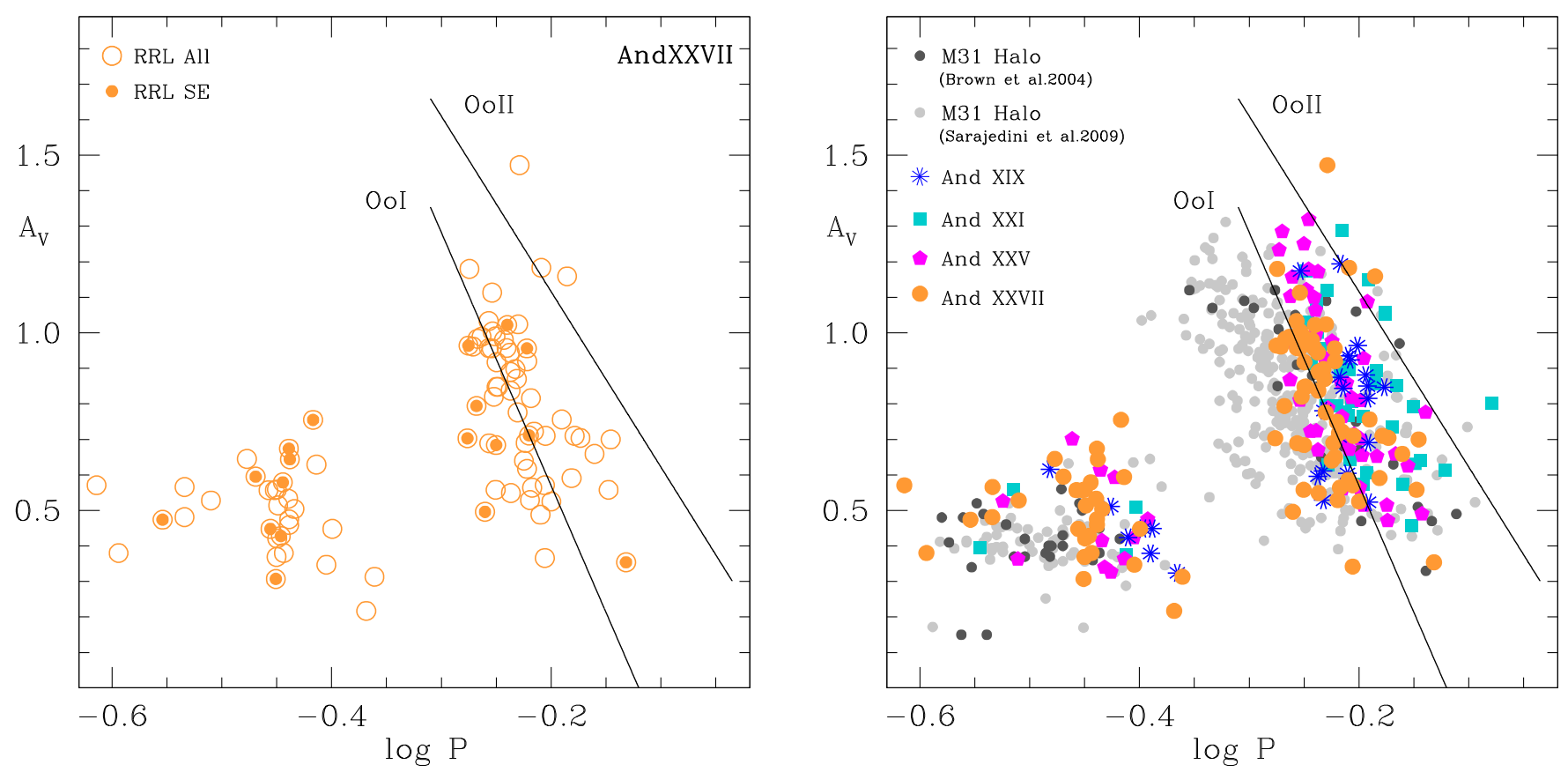

Figure 5. Left: period-amplitude diagram of the RR Lyrae stars in the field of And XXVII (orange circles). The filled orange circles are stars located in the SE region (see Section 4). The two solid lines show the loci defined by Oo I and Oo II RR Lyrae stars, following Clement \& Rowe (2000). Right: period-amplitude diagram of the RR Lyrae stars in the field of And XXVII (orange filled circles) compared with the RR Lyrae stars in And XIX (blue asterisks, from Paper I), And XXI (cyan squares, from Paper II), And XXV (magenta pentagons, from Paper III), and in three HST fields in the M31 halo from Sarajedini et al. (2009; gray dots) and Brown et al. (2004; dark gray dots), respectively.

Table 3

Properties of Selected Samples of RR Lyrae Stars in And XXVII

\begin{tabular}{|c|c|c|c|c|c|c|c|}
\hline id & $N(\mathrm{RRab}+\mathrm{RRc})$ & $\langle V(R R)\rangle$ & $\left\langle P_{\mathrm{ab}}\right\rangle$ & $\left\langle P_{\mathrm{c}}\right\rangle$ & $E(B-V)$ & $(m-M)_{0}$ & $f_{c}$ \\
\hline ALL & $58+31$ & $25.15 \pm 0.17$ & $0.59 \pm 0.05$ & $0.35 \pm 0.04$ & $0.04 \pm 0.05$ & $24.55 \pm 0.26$ & 0.35 \\
\hline SE & $9+9$ & $25.17 \pm 0.17$ & $0.58 \pm 0.05$ & $0.35 \pm 0.03$ & $0.05 \pm 0.05$ & $24.54 \pm 0.26$ & 0.50 \\
\hline SE1 & $6+9$ & $25.24 \pm 0.06$ & $0.55 \pm 0.01$ & $0.35 \pm 0.03$ & $0.03 \pm 0.05$ & $24.67 \pm 0.20$ & 0.60 \\
\hline $\mathrm{C}$ & $6+5$ & $25.15 \pm 0.15$ & $0.62 \pm 0.03$ & $0.33 \pm 0.05$ & $0.07 \pm 0.05$ & $24.46 \pm 0.25$ & 0.45 \\
\hline NORTH & $29+15$ & $25.09 \pm 0.16$ & $0.60 \pm 0.04$ & $0.36 \pm 0.04$ & $0.05 \pm 0.03$ & $24.46 \pm 0.22$ & 0.35 \\
\hline SOUTH & $29+16$ & $25.20 \pm 0.15$ & $0.59 \pm 0.05$ & $0.34 \pm 0.04$ & $0.03 \pm 0.06$ & $24.63 \pm 0.27$ & 0.35 \\
\hline
\end{tabular}

displayed. The CMD appears to be heavily contaminated by MW foreground stars in the red part $(1.4 \leqslant B-V \leqslant 1.9 \mathrm{mag})$ and by background unresolved galaxies in the blue part $(-0.1 \leqslant B-V \leqslant 0.1 \mathrm{mag}$, see Section 9 in Paper I, and e.g., Bellini et al. 2010; Bellazzini et al. 2011). In the right panel plot, the red filled circles are RRab stars (58 sources), the blue open circles are RRc stars (31 sources), and the green triangle is the AC. The RR Lyrae stars trace the HB; their distribution in apparent $V$ mean magnitude is very broad, ranging from 24.70 to $25.55 \mathrm{mag}$ (see Figure 10). A most significant peak is observed at $\langle V(R R)\rangle \sim 25.25 \mathrm{mag}$, followed by a second peak at $\langle V(R R)\rangle \sim 25.10 \mathrm{mag}$.

The four panels in Figure 11 show the CMDs of sources in the four individual CCDs of the LBC mosaic (labeled C1, C2, C3, and C4). Similarly, Figure 12 shows the distributions in apparent $V$ mean magnitude of the RR Lyrae stars in $\mathrm{C} 1, \mathrm{C} 2$, $\mathrm{C} 3$, and $\mathrm{C} 4$, separately. Although contamination by foreground MW stars/background unresolved galaxies (see the left panel of Figure 9) and broadening of both RGB and HB are present in each of the four individual CMDs, C3 shows a more prominent RGB extending as bright as $V \sim 22.5 \mathrm{mag}$, whereas the RGB in the other three CCDs seems to be truncated around $V \sim 23.5$ mag. Furthermore, as shown by Figure 12 the peak at $\langle V(R R)\rangle \sim 25.25 \mathrm{mag}$ is mainly due to the RR Lyrae stars located in $\mathrm{C} 3$.

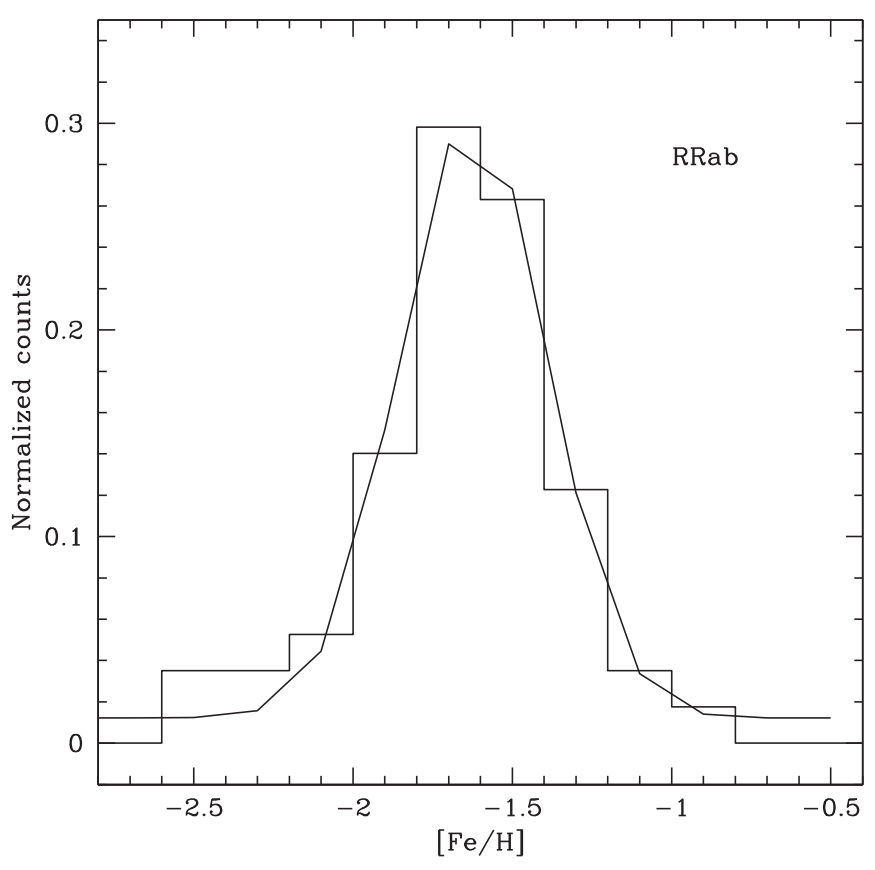

Figure 6. Metallicity distribution of And XXVII RRab stars obtained using Alcock et al.'s (2000) method. The distribution peaks at $[\mathrm{Fe} / \mathrm{H}] \sim-1.62$, with $\sigma \sim 0.23$ dex. 


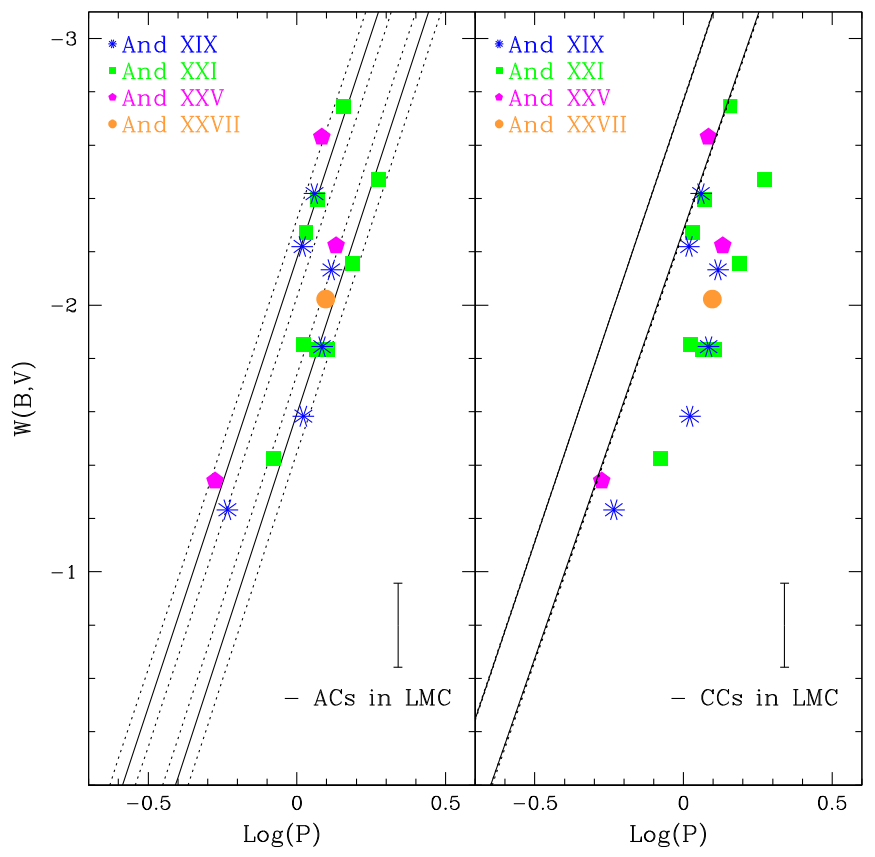

Figure 7. Position on the $P W$ plane of star V2 (orange filled circle). The solid lines represent the $P W$ relations for ACs (left panel; Ripepi et al. 2014) and CCs in the LMC (right panel; Jacyszyn-Dobrzeniecka et al. 2016), respectively. The blue, green, and magenta symbols are ACs we have identified in And XIX (Paper I), And XXI (Paper II), and And XXV (Paper III). The dotted lines show the $\pm 1 \sigma$ deviations. For the CCs, the errors of the fits are very small and the confidence contours are very close to the fits.

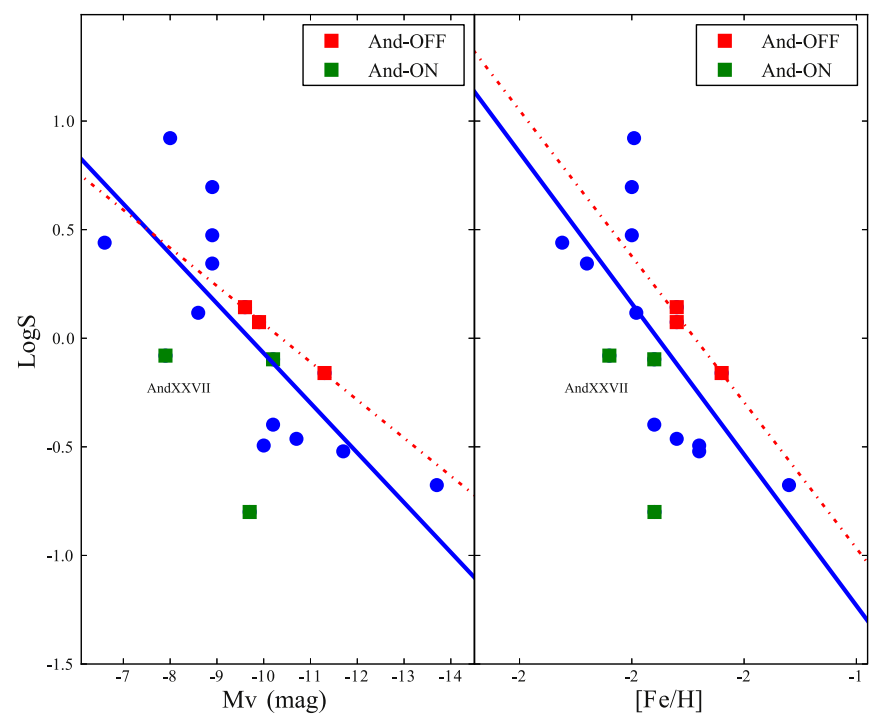

Figure 8. Specific frequencies of ACs in the dwarf satellite galaxies of M31 and the MW vs. luminosity (left panel) and metallicity (right panel) of the host systems. The blue filled circles are MW dwarfs. The red and green filled squares are M31 satellites off and on the GPoA, respectively.

To investigate the spatial distribution of And XXVII stars we selected objects in the RGB region of the CMD obtained in the total LBC FoV (see the left panel of Figure 9) with magnitude and color in the ranges of $22.5 \leqslant V \leqslant 25.3 \mathrm{mag}$ and $0.8 \leqslant B-V \leqslant 1.3 \mathrm{mag}$, respectively. We built isodensity maps by binning these RGB stars in $1 ! 2 \times 1$ ! 2 boxes and smoothing with a Gaussian kernel of $1 ! 2(0.02)$ FWHM. The left panel of Figure 13 shows the RGB isodensity maps where the outermost contour levels are $3 \sigma$ above the sky background.

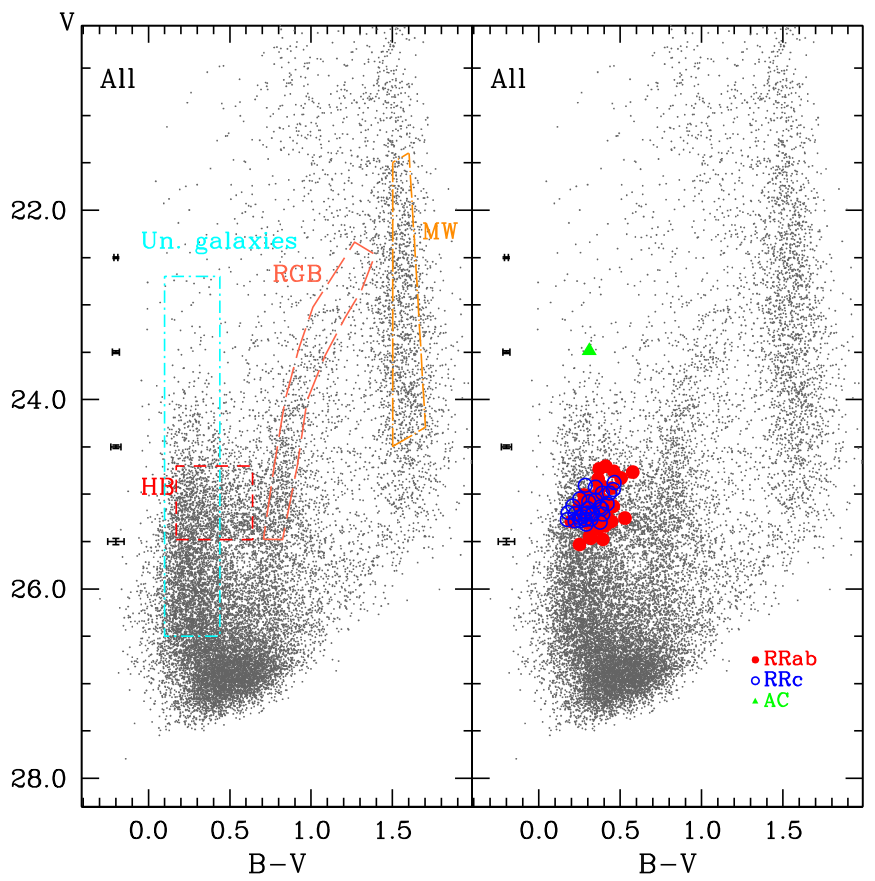

Figure 9. Left: CMD of the sources in the whole LBC FoV. Only objects with $-0.35 \leqslant$ sharpness $\leqslant 0.35$ and $\chi<1.5$ are displayed. The RGB, HB, and MW selections are marked by the dashed regions. Right: same as the left panel but with superimposed the variable stars. The red filled circles are the RRab stars, the blue open circles are the RRc stars, and the green triangle is the AC.

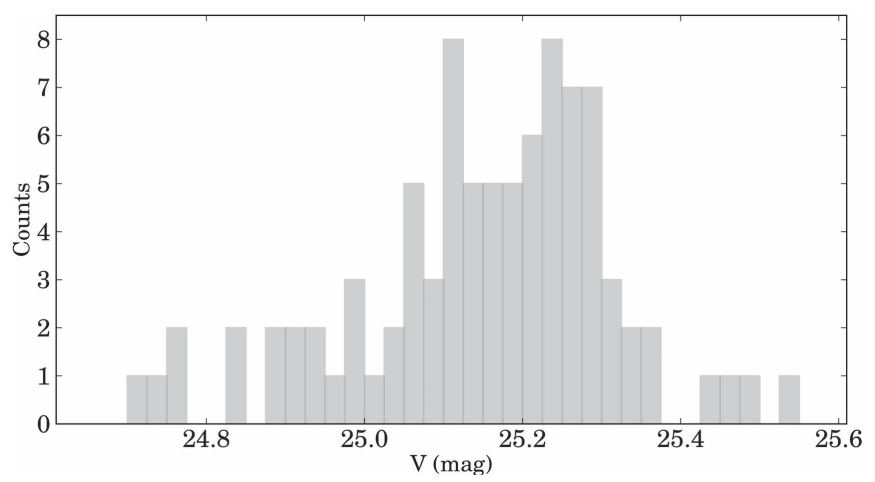

Figure 10. Distribution in apparent $V$ mean magnitude of the 89 RR Lyrae stars identified in the field of And XXVII. The bin size is $0.025 \mathrm{mag}$.

Interesting features are revealed by the isodensity contours of this selection. A high-count isodensity contour shows up around Richardson et al.'s (2011) center coordinates of And XXVII. This region, named $\mathrm{C}$ in the figure, has been marked with a dotted circle of 4 arcmin in radius that is entirely contained within $\mathrm{C} 2$. The second isodensity has a very elongated structure that extends in the southeast direction and reaches a second high-count isodensity displaced by about 0.2 in the southeast direction from the center of the $\mathrm{C}$ region. This second region has been named SE and is marked by a dotted circle of 4 arcmin in radius with center coordinates: R.A. $=00^{\mathrm{h}} 38^{\mathrm{m}} 10^{\mathrm{s}} 4, \quad$ decl. $=+45^{\circ} 21^{\prime} 34^{\prime \prime}$ that is entirely contained within $\mathrm{C} 3$. In both regions, $\mathrm{SE}$ and $\mathrm{C}$, we adopted a radius of 4 arcmin that defines an area twice the high-count isodensity contours. In the $\mathrm{SE}$ region there is a concentration of stars as high as that in the $\mathrm{C}$ region and a higher number of $\mathrm{RR}$ Lyrae stars. 


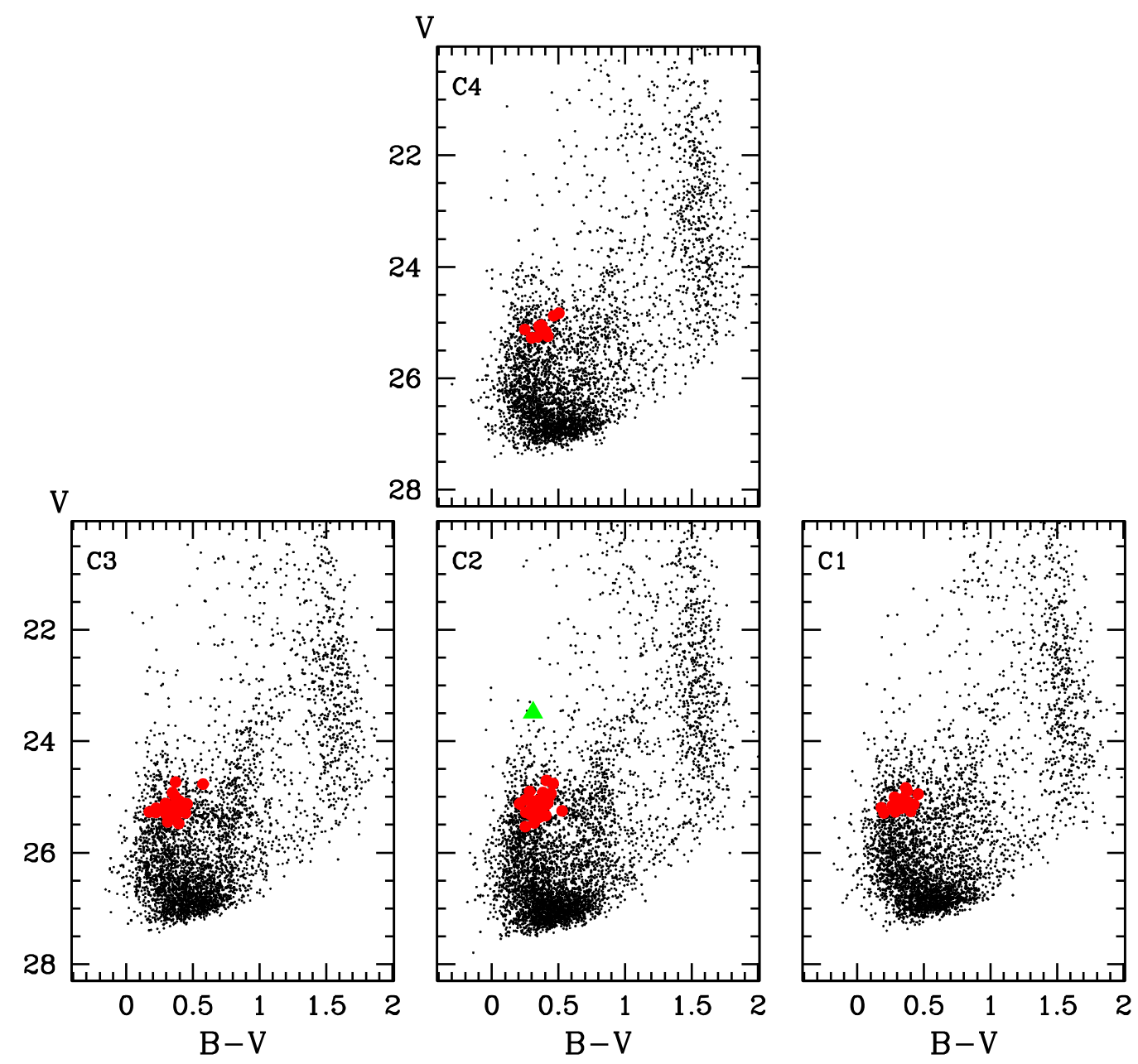

Figure 11. CMDs of sources in the four CCDs of the LBC mosaic. Only objects with $-0.35 \leqslant$ sharpness $\leqslant 0.35$ and $\chi<1.5$ are displayed. The red filled circles are the RR Lyrae stars: 32 in C3, 29 in C2, 21 in C1, and 7 in C4. The green triangle is the AC that is located in C2.

The presence of two high-density contours on the map of Figure 13 points out that And XXVII has a very complex physical structure. We have to stress that the contamination of background and foreground sources can hamper the RGB stellar counts even if the selection was performed in the specific region of the CMD-containing $\mathrm{RGB}$ stars at the distance of And XXVII (see left panel of Figure 9). Moreover, a complex physical structure for And XXVII is also corroborated by the wide RGB and the large spread in mean apparent magnitude of the RR Lyrae stars $(\langle V(R R)\rangle)$ that can be attributed a distance spread. Three possible alternative scenarios to explain And XXVII's structure and its two stellar concentrations are presented in Section 7. Isodensity contours were also computed by selecting stars in the HB region $(25.6 \leqslant V \leqslant 24.6 \mathrm{mag}$, $0.17 \leqslant B-V \leqslant 0.64 \mathrm{mag}$ ) of And XXVII CMD, as shown in the right panel of Figure 13. The HB region of the CMD is contaminated by unresolved background galaxies (blue box, left panel of Figure 9), but still the HB stars follow the structure traced by the RGB stars. We tried to select And XXVII's HB stars by choosing objects closer to the CMD region defined by the RR Lyrae stars, but given the presence in this region of the CMD of unresolved background galaxies, the exact shape of the HB isodensities depends on the selection performed. For the RGB selection, the contamination is not as crucial as it is for HB stars; indeed if we make multiple selections of the RGB region, the shape of the isodensity contours does not change significantly. In order to characterize the stellar populations in the two isodensities with the highest stellar counts we drew the CMDs of the sources in the C and SE regions separately. The result is shown in Figure 14. The CMD of the SE region is similar to the CMD of $\mathrm{C} 3$ and shows a well-extended, better-defined, and narrower RGB especially around the tip, than the CMD of the C region where RGB instead appears truncated around $V \sim 23.5$ mag, as does the CMD of C2. The HB of SE is also better-defined than that in region $\mathrm{C}$. The lower panel of Figure 15 shows the distribution in apparent $V$ mean magnitude of the 11 RR Lyrae stars in region $\mathrm{C}$, while the upper panel shows the mean magnitude distribution of the $18 \mathrm{RR}$ Lyrae stars in region SE. Of the 18 RR Lyrae stars, 15 contained in the SE region trace a rather tight $\mathrm{HB}$ with $\langle V(R R)\rangle=25.24 \mathrm{mag}$ and $\sigma=0.06 \mathrm{mag}$ (on average 15 stars). On the contrary, the 11 RR Lyrae stars of region $\mathrm{C}$ are rather spread in magnitude (see the left panel of Figure 14 and bottom panel of Figure 15) with $\langle V(R R)\rangle=25.15 \mathrm{mag}$ and $\sigma=0.15$ mag. This makes us wonder whether the actual center of And XXVII might be in the SE region rather than in $\mathrm{C}$, as found by Richardson et al. (2011).

We have superimposed on the SE CMD an isochrone of $13 \mathrm{Gyr}$ with $[\mathrm{Fe} / \mathrm{H}]=-1.8$ as derived from the web interface CMD $2.9^{10}$

\footnotetext{
${ }^{10}$ http://stev.oapd.inaf.it/cgi-bin/cmd
} 

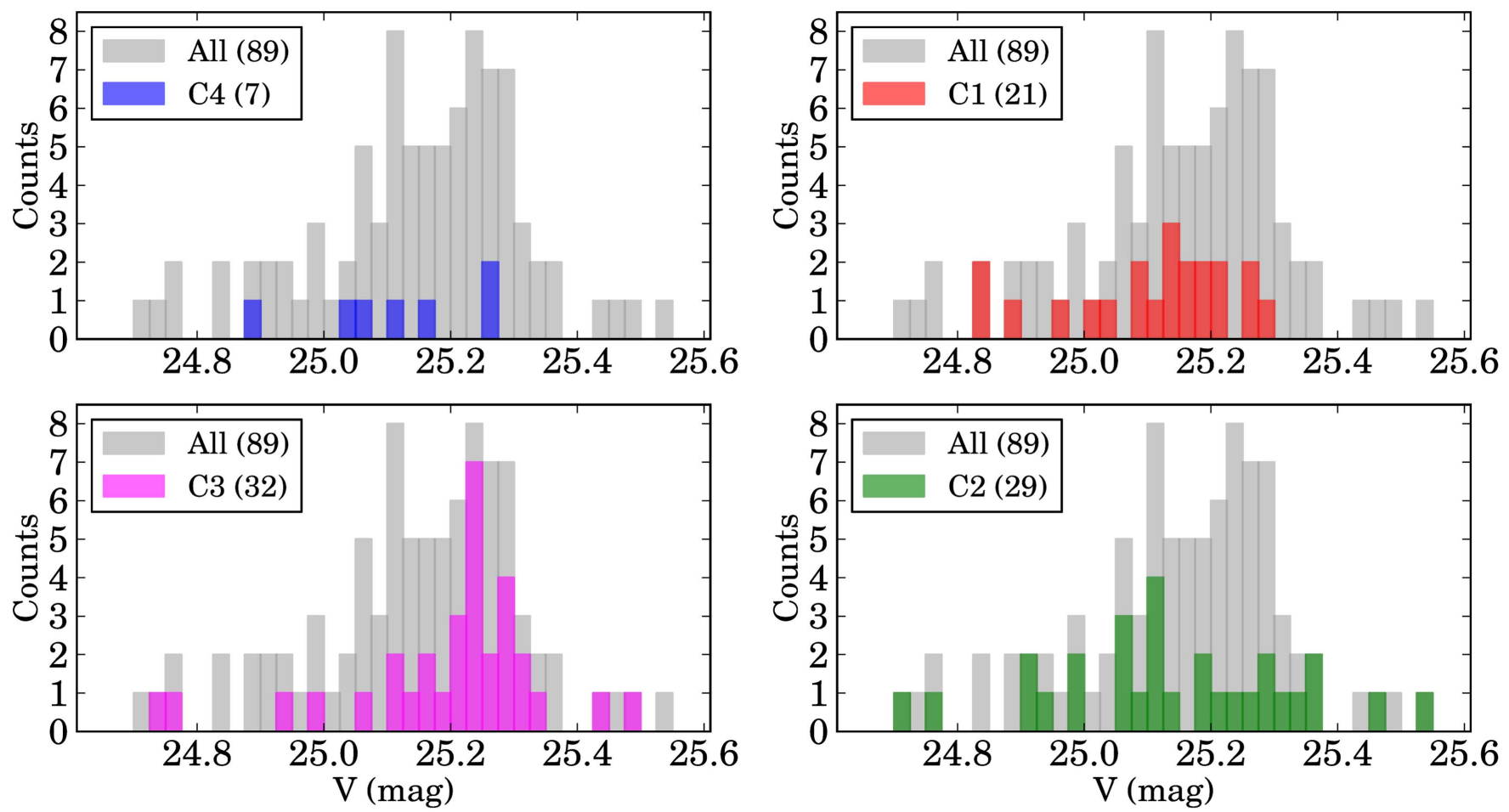

Figure 12. Distribution in apparent $V$ mean magnitude of the RR Lyrae stars in the four CCDs of the LBC mosaic. The bin size is 0.025 mag.
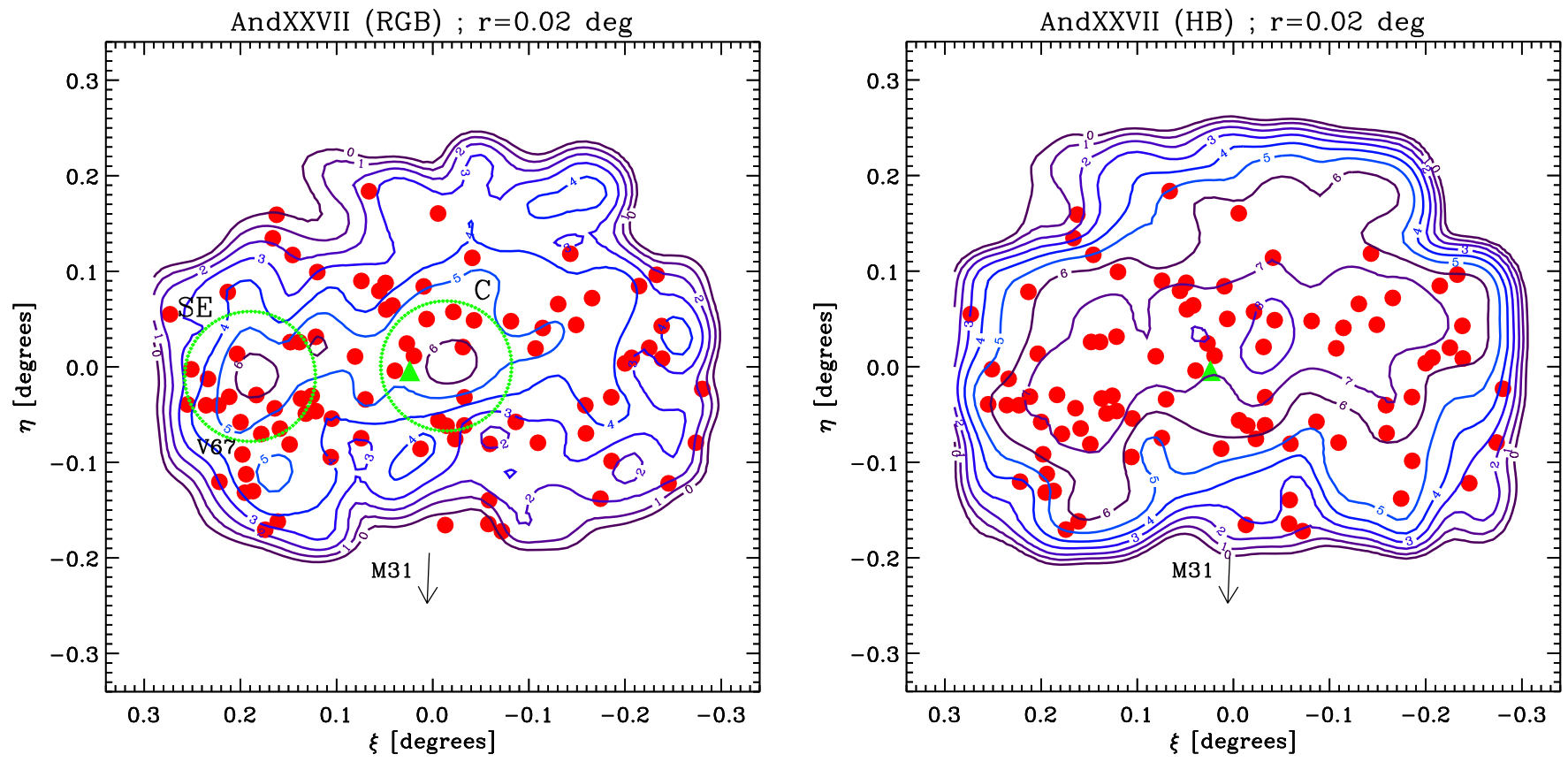

Figure 13. Left: isodensity contours of RGB stars in And XXVII. The $X$ and $Y$ axes are, respectively, R.A. and decl. differential coordinates computed from the center of the galaxy given by Richardson et al. (2011). The red filled circles are RR Lyrae stars, and the green filled triangle is the AC. The RR Lyrae star for which we have estimated a metallicity of $[\mathrm{Fe} / \mathrm{H}]=-1.86 \sim 0.50 \mathrm{dex}$ (on the Carretta et al. 2009 scale) from the Fourier parameter $\phi_{31}$, is labeled V67. The green circles mark areas of 4 arcmin in radius around regions $\mathrm{C}$ and SE, respectively. Right: same as the left panel but for And XXVII HB stars.

based on the Marigo et al. (2017) evolutionary tracks. The isochrone was corrected for the distance and reddening derived in Section 5 for the SE region. This isochrone well fits the position of RR Lyrae and RGB stars. The comparison between the isochrone and observed CMD suggests a higher metallicity value for And XXVII than was measured by Collins et al. (2013). The isochrone fit result is consistent (within the errors) with the metallicity estimated using the RR Lyrae stars in Section 3.2. A similar conclusion is also reached by comparison with the RGB and $\mathrm{HB}$ ridge lines of galactic Globular Clusters (GCs) with different metallicities; the best result is obtained for NGC 4147 with $[\mathrm{Fe} / \mathrm{H}]=-1.8$ dex (Harris 1996, 2010 edition). In the following we adopt $[\mathrm{Fe} / \mathrm{H}]=-1.8$ dex for the metallicity of And XXVII. 


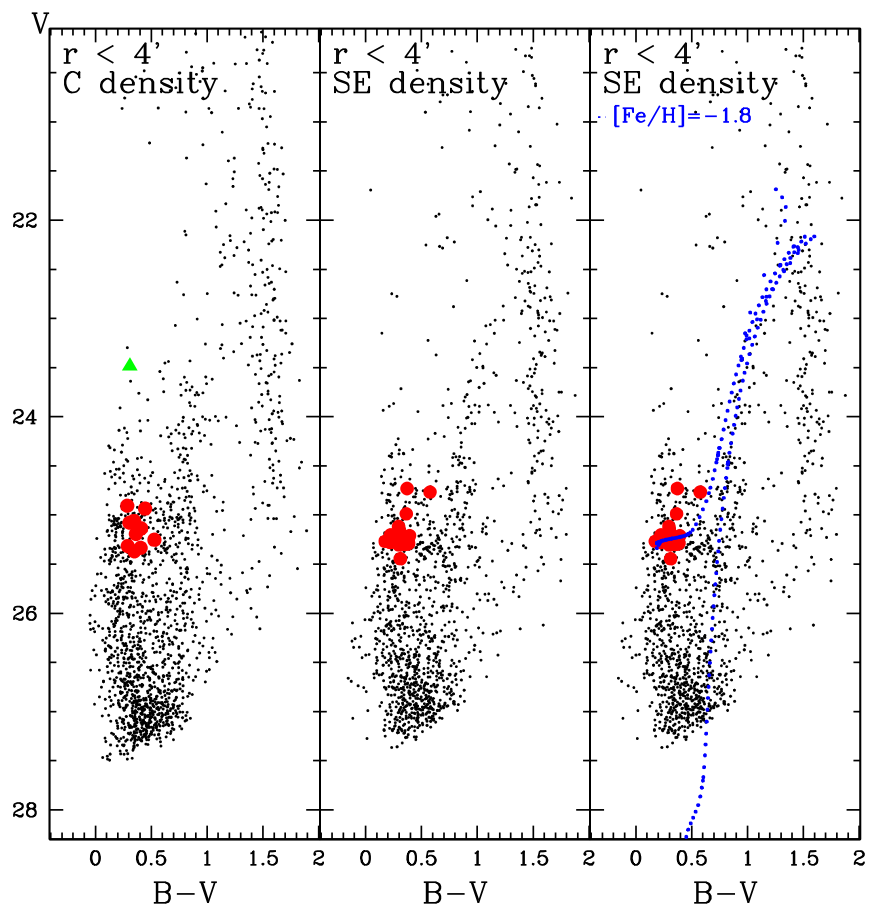

Figure 14. Left: $\mathrm{CMD}$ of sources in the $\mathrm{C}$ overdensity region. Center: same as the left panel, but for the SE region. Right: CMD of the SE region with a superimposed isochrone of $13 \mathrm{Gyr}$ with $[\mathrm{Fe} / \mathrm{H}]=-1.8$ dex (blue dotted line; see the text for details).
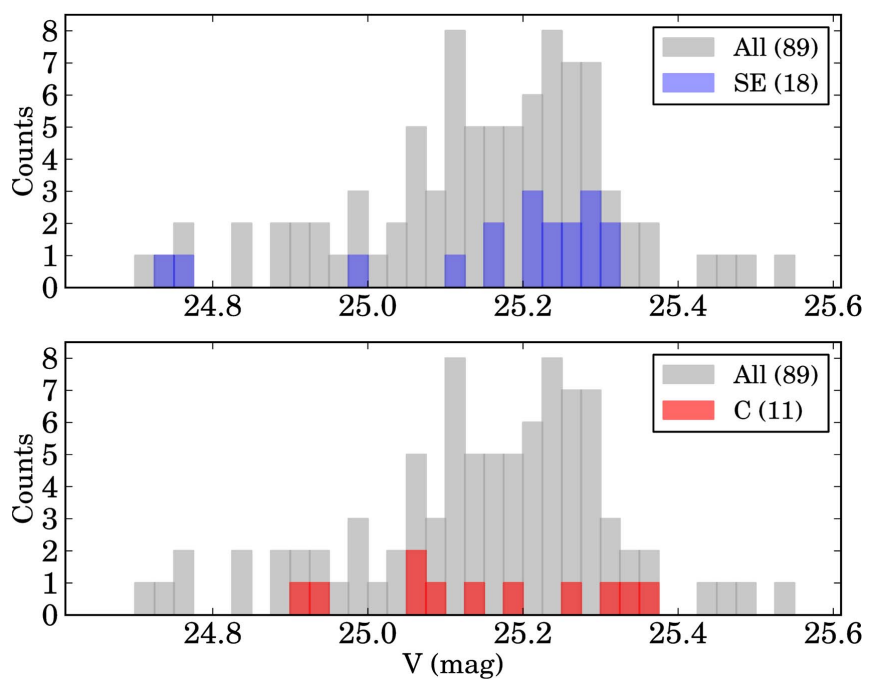

Figure 15. Lower panel: distribution in apparent $V$ mean magnitude of the 11 RR Lyrae stars in the $\mathrm{C}$ region. Upper panel: same as the lower panel, but for the 18 RR Lyrae stars in the SE region.

\section{Distance and Structure}

The mean luminosity of the RR Lyrae stars can be used to estimate the distance to And XXVII. We are aware that some of the RR Lyrae stars in the field of And XXVII can belong to the M31 halo, but since it is not possible to distinguish them, in deriving the distance we used the sample of 89 RR Lyrae stars found in this work. The average $V$ apparent magnitude of the 89 RR Lyrae stars in the field of And XXVII is $\langle V(R R)\rangle=25.15 \mathrm{mag}$ ( $\sigma=0.17 \mathrm{mag}$ average on 89 stars $)$. As in previous papers of this series, we derived the reddening from the RR Lyrae stars by adopting the method of Piersimoni et al. (2002, equation on page 1538). The reddening estimated in this way, $\langle E(B-V)\rangle=0.04 \pm 0.05 \mathrm{mag}$ (where the error is the standard deviation of the mean), is slightly smaller but still consistent within $1 \sigma$ with the value inferred by Schlegel et al. (1998, $E(B-V)=0.08 \pm 0.06 \mathrm{mag})$. The visual absorption $A_{V}$ was derived using the extinction law $A_{V}=3.1 \times E(B-V)$ from Cardelli et al. (1989). We then adopt $M_{\mathrm{V}}=0.54 \pm 0.09 \mathrm{mag}$ for the absolute visual magnitude of RR Lyrae stars with a metallicity $[\mathrm{Fe} / \mathrm{H}]=-1.5$ dex (Clementini et al. 2003) and $\frac{\Delta M_{V}}{\Delta[\mathrm{Fe} / \mathrm{H}]}=-0.214 \pm 0.047 \mathrm{mag} / \mathrm{dex}$ (Clementini et al. 2003; Gratton et al. 2004) for the slope of the RR Lyrae luminosity-metallicity relation. For the metallicity of And XXVII we adopt $[\mathrm{Fe} / \mathrm{H}]=-1.8 \pm 0.3$ dex, as derived from isochrone-fitting and consistent with the estimate from the RR Lyrae stars. The resulting distance modulus is $(m-M)_{0}=24.55 \pm 0.26 \mathrm{mag}$. This is $0.16 \mathrm{mag}$ fainter but still consistent, within the large error, with Richardson et al.'s (2011) lower limit of $\geqslant 757 \pm 45 \mathrm{kpc}$ (corresponding to $\left.(m-M)_{0}=24.39 \pm 0.13 \mathrm{mag}\right)$ and placing And XXVII within the M31 complex. The faintest estimate by Conn et al. (2012): $(m-M)_{0}=25.49_{-1.03}^{+0.07} \mathrm{mag}$ has a very large asymmetric error that is likely due to the difficulty in identifying the tip of RGB, which is very scarcely populated in And XXVII. In any case, our estimate is consistent within the errors with the lower limit of Conn et al.'s (2012) estimate. The large error in our distance estimate is due to the significant dispersion of the average magnitude of the whole RR Lyrae star sample. As anticipated in Section 4, And XXVII appears to be in the process of tidal disruption; this may have resulted in the RR Lyrae stars being located at different distances from us, thus causing the large dispersion in $\langle V(R R)\rangle$. To further investigate the galaxy structure we have compared the number and properties of the $R R$ Lyrae stars located in different parts of the LBC FoV, starting from the two regions with the highest stellar counts, namely regions $\mathrm{C}$ and $\mathrm{SE}$ in Figure 13.

There are 18 RR Lyrae stars in the SE region and only 11 in the $\mathrm{C}$ region. This difference remains even if we reduce the radii of the two regions: in a circular portion of the $\mathrm{SE}$ region of 3 arcmin in radius there are 8 RR Lyrae stars to compare with 6 $\mathrm{RR}$ Lyrae contained in a similar portion of region $\mathrm{C}$. The average $\mathrm{V}$-magnitude, reddening, and other characteristics (total number of RR Lyrae stars, average RRab period, distance modulus, and fraction of RRc pulsators) for the RR Lyrae stars in regions $\mathrm{C}$ and $\mathrm{SE}$ separately are provided in Table 3. Two sets of values are listed for region SE, the first one corresponding to the whole set of 18 variables, and the second one (labeled SE1 in the table) considering only the 15 RR Lyrae stars whose mean magnitude peaks around $\langle V\rangle \sim 25.24$ mag. The distance moduli of $\mathrm{C}$ and SE differ by 0.08 mag in the first case; this difference is still within $1 \sigma$ given the large dispersion of the estimates. The difference is of 0.21 mag in the second case (SE1) and may be due to a real radial distance effect. However, considering the large errors we are dealing with, this conclusion has to be taken with caution. The spread in magnitude can be due to a real three-dimensional (3D) effect, but not as big as $0.21 \mathrm{mag}$. The centers of regions $\mathrm{C}$ and SE are $\sim 0.2$ apart in the sky, which, at the distance of And XXVII, corresponds to a physical projected separation of $\sim 3 \mathrm{kpc}$. Is this an indication that the galaxy is disrupted and the SE component is $3 \mathrm{kpc}$ away from the galaxy center? Or, since SE has the cleanest CMD and the largest concentration of RR Lyrae stars, most of which are located approximately, at the 


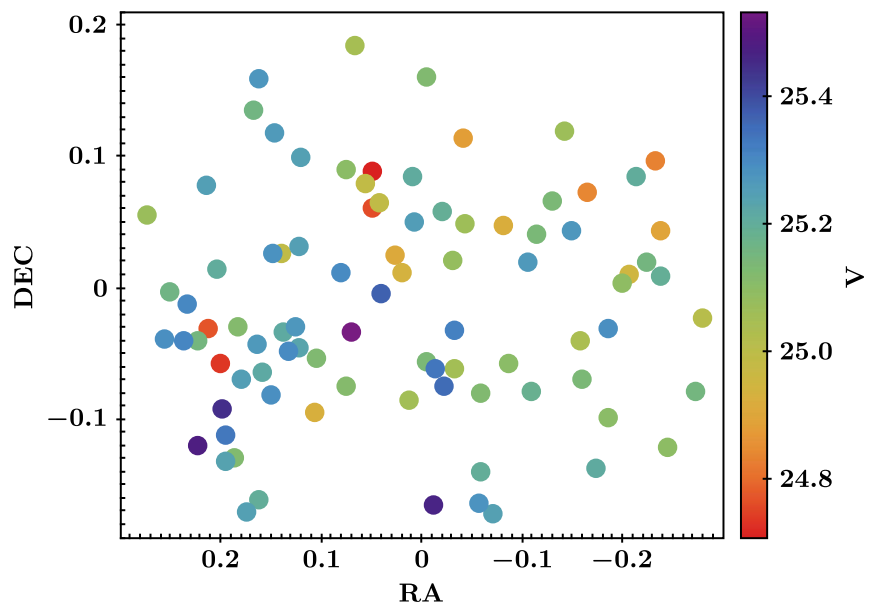

Figure 16. Spatial distribution of the RR Lyrae stars in the field of And XXVII. The stars are color-coded according to their mean $V$ magnitudes.

same distance from us, could SE be the remaining nucleus of the now disrupted And XXVII?

Triggered by these two different possibilities, we divided the RR Lyrae stars into two samples, corresponding, respectively, to the south and north portions of And XXVII according to Richardson et al.'s (2011) center coordinates. The characteristics of the RR Lyrae stars in the north and south samples are also summarized in Table 3. The north and south distance moduli differ by $\sim 0.2 \mathrm{mag}$, which, although still consistent within the respective errors, could indicate a 3D effect where the north part of And XXVII is closer to us than the south part.

We also tested whether possible zero-point differences among the four CCDs of the LBC might cause the observed differences, by comparing the median apparent $B$ and $V$ magnitudes of objects in the blue part $(-0.1 \leqslant B-V \leqslant 0.1$ mag, $24 \leqslant V \leqslant 26 \mathrm{mag})$ and in the red part $(1.6 \leqslant B-V \leqslant 1.9$ mag, $23 \leqslant V \leqslant 25 \mathrm{mag}$ ) of the CMD.

The differences among the four CCDs range from 0.001 to 0.03 mag both in the $B$ - and $V$-bands, hence we cannot explain the difference observed between the average apparent magnitudes of the north and south RR Lyrae subsamples. Similarly, these differences cannot be explained by differential reddening, as reddening is generally small and very similar among the various RR Lyrae subsamples. We also computed the average reddening derived from the RR Lyrae stars in the four CCDs, obtaining: $\langle E(B-V)\rangle_{C 1}=0.03 \pm 0.06 \mathrm{mag}, \quad\langle E(B-V)\rangle_{C 2}=0.03 \pm$ $0.06 \mathrm{mag}, \quad\langle E(B-V)\rangle_{C 3}=0.05 \pm 0.05 \mathrm{mag}$, and $\langle E(B-$ $V)\rangle_{C 4}=0.01 \pm 0.06 \mathrm{mag}$. Therefore, we conclude that the magnitude differences are real and likely due to a 3D effect. Figure 16 shows the spatial distribution of the 89 RR Lyrae stars in the field of And XXVII, where each source is color-coded according to its mean apparent magnitude $(\langle V\rangle)$. If we assume that differences in $\langle V\rangle$ values are totally due to a projection/distance effect, which is sensible given the similarity of pulsation properties of the 89 RR Lyrae stars and the low and homogeneous reddening in the area, the distribution in Figure 16 suggests that And XXVII's center likely is in the SE overdensity and the galaxy is tilted, with the northwest portion being closer to us than the southeast part.

We recall that among the three other M31 satellites we observed with the LBT, we did not find evidence of a particular smearing in mean magnitude of the RR Lyrae stars in And XIX and And XXV, whereas the magnitude spread observed in And XXI was found to be fully justified by the presence of two RR Lyrae populations with different metallicities, as also suggested by the bimodal period distribution of the RRab stars (see Figure 2 in Paper II). However, the scatter of the RR Lyrae star mean magnitude in the field of And XXVII is over twice that observed in And XXI, while there is no evidence of bimodality in the periods of the RRab stars. This, along with the large spread in the spatial distribution of the RR Lyrae population, leads us to conclude that either we are resolving the 3D structure of a galaxy in the final stage of disruption or we are sampling the RR Lyrae stars in And XXVII together with those in a background/foreground structure like the M31 NW stream or the M31 halo.

\subsection{Different Old Stellar Populations?}

If the differences in magnitudes of the RR Lyrae star subsamples are not due to distance effects, a possible alternative explanation is the presence in And XXVII of different old stellar generations. To test this hypothesis, the positions on the CMD of the different samples of RR Lyrae stars listed in Table 3 were compared to the model of Marconi et al. (2015) and the $13 \mathrm{Gyr}$ isochrones of Marigo et al. (2017) after correction for the reddening and the distance modulus derived using our whole RR Lyrae stars sample $\left[(m-M)_{0}=24.55 \pm 0.26\right.$ mag, $\langle E(B-V)\rangle=0.04 \pm 0.05 \mathrm{mag}]$. To further constrain the observations we also compared the observed period distribution of the RRab pulsators to the models. Starting from the C sample, the position on the CMD and the period distribution of the RRab can be reproduced by the models with metallicities from $[\mathrm{Fe} / \mathrm{H}]=-2.3$ to -1.5 dex, $M=0.67-0.8 \quad M_{\odot}, \quad T_{\text {eff }}=$ 5900-6900 K, and $\log L=1.69-1.79 L_{\odot}$. The $[\mathrm{Fe} / \mathrm{H}]=-1.5$ models are at the limit of the redder RR Lyrae stars.

The SE sample appears on average bluer and fainter than the best matching models with metallicities from $[\mathrm{Fe} / \mathrm{H}]=-2.3$ to $-1.7 \mathrm{dex}, M=0.716-0.8 M_{\odot}, T_{\text {eff }}=6000-6900 \mathrm{~K}$ and $\log L=1.72-1.76 L_{\odot}$. Moreover, the observed distribution in periods of the SE RRab sample is shorter than the one derived from the latter models. Indeed, models more metal-rich than $[\mathrm{Fe} / \mathrm{H}]=-1.8$ are too red to reproduce the color of the RRab stars. The observations are only matched if we correct the models for the distance modulus and reddening found for the SE1 sample $\left[(m-M)_{0}=24.67 \pm 0.26 \mathrm{mag},\langle E(B-V)\rangle=\right.$ $0.03 \pm 0.05 \mathrm{mag}]$. We concluded that a scenario in which the RR Lyrae stars have the same metallicities, but different distances, is best matched by the models as shown in the right panel of Figure 17. In this case both the RR Lyrae stars and the RGB stars are well reproduced by the $13 \mathrm{Gyr}$ isochrones, with a $[\mathrm{Fe} / \mathrm{H}]=-1.8$ corrected for the distance moduli $(m-M)_{0}=24.46 \mathrm{mag}$ and $(m-M)_{0}=24.67 \mathrm{mag}$ that represents the extreme values reported in Table 3 . The twofold RR Lyrae population can thus not be explained by the presence of two generations of stars with different metallicities at the same distance. The simple old stellar generation placed at different distances has to be preferred over the double stellar population (see the right panel of Figure 17). We are perhaps sampling two different regions in the M31 halo, one belonging to And XXVII and the other to the NW stream that is in the back/front of And XXVII (see Section 6). 

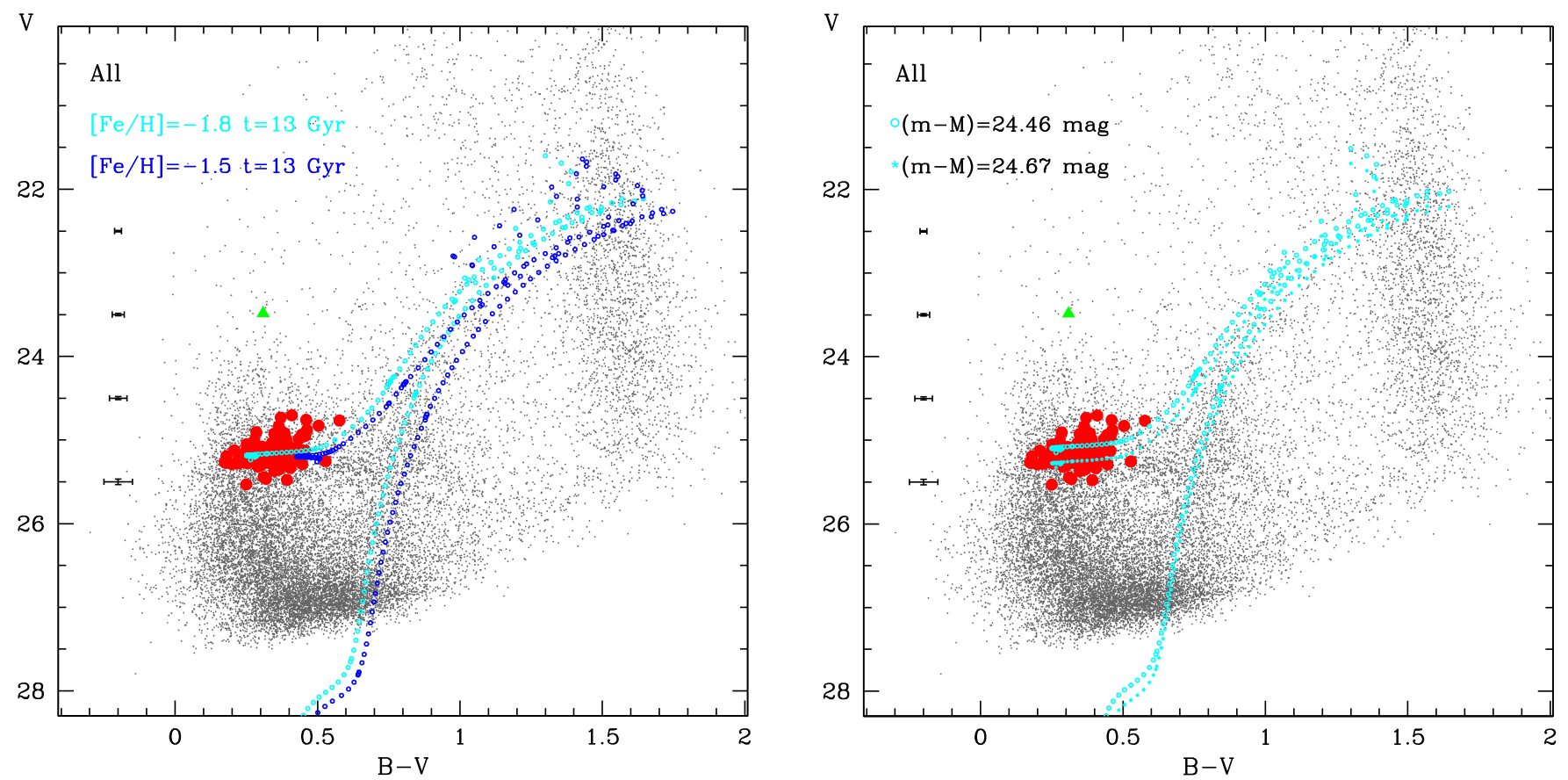

Figure 17. Left: same as Figure 9 with superimposed $13 \mathrm{Gyr}$ isochrones from Marigo et al. (2017), with $[\mathrm{Fe} / \mathrm{H}]=-1.8 \mathrm{dex}(\mathrm{cyan}$ line) and $[\mathrm{Fe} / \mathrm{H}]=-1.5$ dex (blue line). Right: same as the left panel, but with the same $13 \mathrm{Gyr}$ isochrone and $[\mathrm{Fe} / \mathrm{H}]=-1.8$ corrected for distance moduli of $(m-M)=24.46 \mathrm{mag}$ (upper line) and $(m-M)=24.67 \mathrm{mag}$ (lower line), respectively.

Table 4

Properties of the Variable Stars in the Andromeda Satellite Galaxies

\begin{tabular}{|c|c|c|c|c|c|c|c|}
\hline Name & $N(\mathrm{RRab}+\mathrm{RRc})$ & $\left\langle P_{\mathrm{ab}}\right\rangle$ & $f_{c}$ & $N(\mathrm{AC})$ & $N$ (AC) Confirmed ${ }^{\mathrm{a}}$ & Member (GpoA) & Reference \\
\hline And I & $72+26$ & 0.57 & 0.26 & $1 ?$ & 0 & yes & (1) \\
\hline And II & $64+8$ & 0.57 & 0.11 & 1 & 0 & no & (2) \\
\hline And III & $39+12$ & 0.66 & 0.23 & $5 ?$ & 2 & yes & (1) \\
\hline And XI & $10+5$ & 0.62 & 0.33 & 0 & 0 & yes & (4) \\
\hline And XIII & $12+5$ & 0.66 & 0.30 & 0 & 0 & yes & (4) \\
\hline And XVI & $3+6$ & 0.64 & 0.33 & 0 & 0 & $\mathrm{no}^{\mathrm{b}}$ & $(5),(6)$ \\
\hline And XXV & $46+11$ & 0.60 & 0.19 & 2 & 1 & yes & (9) \\
\hline And XXVII & $58+31$ & 0.59 & 0.35 & 1 & 1 & yes & (10) \\
\hline
\end{tabular}

Notes.

${ }^{\text {a }}$ Confirmed AC, based on the $P W$ relation (see Paper III).

${ }^{\mathrm{b}}$ Offset by $8 \mathrm{kpc}$ from the GPoA (Ibata et al. 2013).

References. (1) Pritzl et al. (2005); (2) Pritzl et al. (2004); (3) Pritzl et al. (2002); (4) Yang \& Sarajedini (2012); (5) A. Mercurio et al. (2017, in preparation); (6) Monelli et al. (2016); (7) Cusano et al. (2013); (8) Cusano et al. (2015); (9) Cusano et al. (2016); (10) this work.

\section{Association with the Northwest Stream}

The NW stream is a stellar structure extending up to $120 \mathrm{kpc}$ from the center of M31, which in its arc-path intersects And XXVII. This stream was first detected by Richardson et al. (2011) in the PandAS map of metal-poor $([\mathrm{Fe} / \mathrm{H}]<-1.4$ dex $)$ RGB stars. The mean surface brightness of the NW stream is 28-29 mag $\operatorname{arcsec}^{-2}$ and the estimated total mass is $\sim 10^{7} M_{\odot}$ (Carlberg et al. 2011). In the literature a mass of $M=8.3_{-3.9}^{+2.8} \times 10^{7} M_{\odot}$ was estimated for And XXVII by Collins et al. (2013) using the velocity dispersion, under the assumption that the galaxy is in dynamical equilibrium and is spherically symmetric. However, both assumptions are very uncertain given the clearly disruptive nature of this galaxy. A very rough mass estimate inferred from the total number of RR Lyrae stars would place And XXVII in the class of $10^{7} M_{\odot}$ dwarf galaxies, like And I or And VI (see Table 4 for the number of RR Lyrae stars in these galaxies and the masses in Collins et al. 2014).

Projected along the NW stream are also seven GCs. Veljanoski et al. (2014) measured their RVs and found that six of them show a clear trend with the projected distance from the M31 center, their velocities becoming more negative as the GCs approach the M31 center. Monte Carlo simulations performed by Veljanoski et al. (2014) showed that these six GCs are physically associated (with an average RV of $-430 \pm 30 \mathrm{~km} \mathrm{~s}^{-1}$ ) and with the NW stellar stream. Given its position along the stream, And XXVII has a RV compatible with that found for the GCs, assuming a quasi-circular inclined orbit where the direction of rotation goes from west to east for the objects in the NW stream. And XXVII may be the satellite 


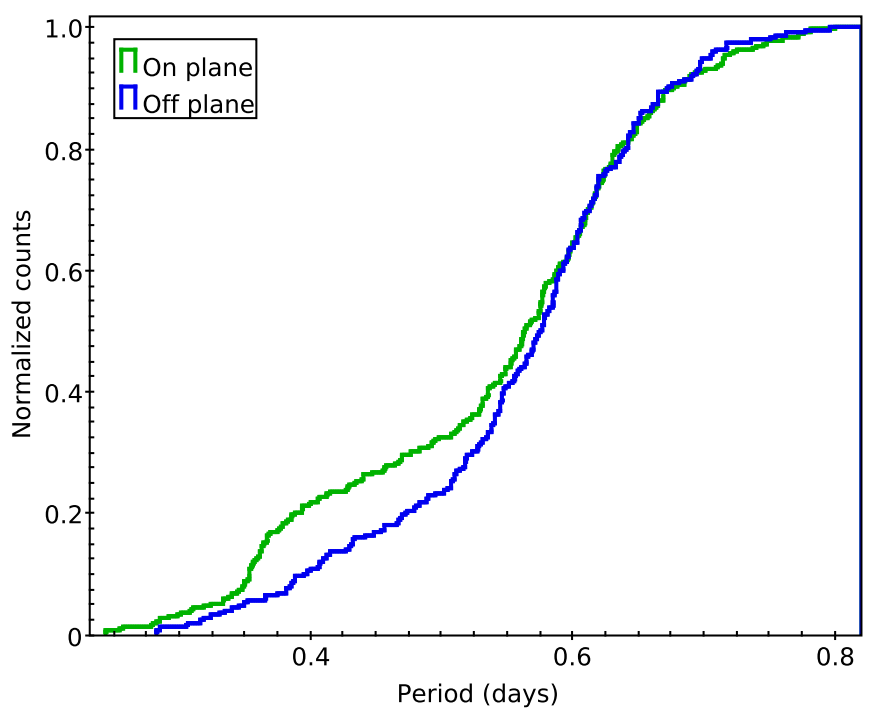

Figure 18. Cumulative period distribution of the RR Lyrae stars in M31 satellite galaxies on and off the GPoA.

galaxy that generated the NW stream. The galaxy is perhaps completing a full orbit around M31 and after a near pericenter passage started losing stars. However, with the current data set we cannot definitively prove this hypothesis. More kinematic and photometric data are needed to investigate the likely connection between the NW stream and And XXVII.

\section{Comparison with Other M31 Satellites and Conclusions}

Following the procedure in Paper III, we compared the pulsation properties of the RR Lyrae populations in the M31 $\mathrm{dSph}$ satellites on and off the GPoA. Table 4 summarizes the characteristics of the pulsating variable stars in the M31 dSphs that have been studied so far for variability, 11 in total. Six of them are on and five are off the GPoA. We computed the average period of the RRab stars for galaxies on and off the plane separately, finding $\left\langle P_{\mathrm{ab}}\right\rangle=0.60 \pm 0.07$ days and $\left\langle P_{\mathrm{ab}}\right\rangle=0.60 \pm 0.06$ days. The fraction of RRc to the total number of RR Lyrae stars is $f_{c}=0.27$ and $f_{c}=0.23$ for on-plane and off-plane satellites, respectively. Hence, both samples are compatible with an Oo-Int classification and show a slight tendency toward the Oo I type as the galaxies get closer to M31 (see also the right panel of Figure 5). The cumulative period distribution of the RR Lyrae stars in the two samples is shown in Figure 18. A two sample Kolmogorov-Smirnov test to check whether there are any differences between the two populations returns a $p$-value of $p=0.03209$, meaning that the two RR Lyrae populations differ significantly. The two samples especially differ in the short-period regime $(P<0.4$ day, see Figure 18). This is mainly due to the large number of shortperiod RR Lyrae stars in And XXVII (indeed the $p$-value in Paper III was $p=0.36$, which is without the RR Lyrae stars of And XXVII). The fraction of RRc stars in And XXVII is oddly high. A similar result was found by Brown et al. (2004) in some fields of the M31 halo. These authors measured a fraction $f_{c}=0.46$, an average period for RRab of $\left\langle P_{\mathrm{ab}}\right\rangle=0.594$ days and an average period for RRc of $\left\langle P_{\mathrm{c}}\right\rangle=0.316$ days. Between Brown et al.'s (2004) results and ours, in And XXVII (see Table 3) there are some similarities like the average period of RRab and the high fraction of RRc. Furthermore, the right panel of Figure 5 shows a similar trend for the period-amplitude diagram of M31 halo and And XXVII RR Lyrae stars. These analogies can be translated into three possible scenarios. (1) a number of the RR Lyrae stars in the field of And XXVII do in fact belong to the M31 halo (the projected distance of And XXVII from the M31 center is $\left.d_{p} \sim 60 \mathrm{kpc}\right)$ and in particular to the NW stream. This would explain the spread in distance we observe from the RR Lyrae stars. (2) And XXVII is not a dwarf galaxy but rather an overdensity in the NW stream (as claimed by Martin et al. 2016) that is largely comprised by M31 halo stars. (3) And XXVII is the progenitor of the NW stream and part of the M31 halo. The high concentration of RR Lyrae stars and the CMD of the And XXVII SE region, along with the possible connection with the GCs in the NW stream seem to support this latter possibility. However, as shown in Figure 9 of Ibata et al. (2014) the M31 halo in the And XXVII neighborhood is very complex and the stars in the metallicity range $-2.5<$ $[\mathrm{Fe} / \mathrm{H}]<-1.7$ dex are placed in streams located all around And XXVII. Investigations of the variable stars and CMD of the area beyond the SE region in the southeast direction, as well as in different regions of the M31 halo and NW stream are essential in order to distinguish between the above different scenarios.

Concerning the frequency of ACs in the M31 satellites, with the discovery of only one AC in And XXVII, we confirm the finding of Paper III that on-plane satellites seem to host only a few or no ACs. There are 4 ACs in total in the 6 on-plane satellites in Table 4, compared with 21 ACs in the 5 off-plane systems. This suggests that only off-plane satellites were able to retain enough gas to give rise to an intermediate-age stellar population and produce ACs. In this regard, the recent detection of an irregularly shaped H I cloud close to but offset from the stellar body of And XIX (Kerp et al. 2016) and of some H I emission displaced by half a degree from And XXI (J. Kerp 2017, private communication), gives support to the possible presence of a 1-2 Gyr-old stellar generation in And XIX and And XXI, and since both these systems are off the GPoA, perhaps in the off-plane M31 satellites in general.

We warmly thank P. Montegriffo for the development and maintenance of the GRATIS software. Financial support for this research was provided by PRIN INAF 2010 (PI: G, Clementini) and by Premiale LBT 2013. The LBT is an international collaboration among institutions in the United States, Italy, and Germany. LBT Corporation partners are The University of Arizona on behalf of the Arizona university system; Istituto Nazionale di Astrofisica, Italy; LBT Beteiligungsgesellschaft, Germany, representing the Max-Planck Society, the Astrophysical Institute Potsdam, and Heidelberg University; The Ohio State University; and The Research Corporation, on behalf of The University of Notre Dame, University of Minnesota, and University of Virginia. We acknowledge the support from the LBT-Italian Coordination Facility for the execution of observations, data distribution, and reduction.

Facility: LBT.

\section{ORCID iDs}

Felice Cusano (iD https://orcid.org/0000-0003-2910-6565 Michele Cignoni ib https://orcid.org/0000-0001-6291-6813 Vincenzo Testa (ii) https://orcid.org/0000-0003-1033-1340 Marcella Marconi (iD https://orcid.org/0000-0002-1330-2927 Vincenzo Ripepi (iD https://orcid.org/0000-0003-1801-426X 


\section{References}

Alcock, C., Allsman, R. A., Alves, D. R., et al. 2000, AJ, 119, 2194 Annibali, F., Nipoti, C., Ciotti, L., et al. 2016, ApJL, 826, L27 Bailey, S. I. 1902, AnHar, 38, 1

Bellazzini, M., Beccari, G., Oosterloo, T. A., et al. 2011, A\&A, 527, A58

Bellini, A., Bedin, L. R., Piotto, G., et al. 2010, A\&A, 513, A50

Brown, T. M., Ferguson, H. C., Smith, E., et al. 2004, AJ, 127, 2738

Bullock, J. S., \& Johnston, K. V. 2005, ApJ, 635, 931

Cacciari, C., Corwin, T. M., \& Carney, B. W. 2005, AJ, 129, 267

Cardelli, J. A., Clayton, G. C., \& Mathis, J. S. 1989, ApJ, 345, 245

Carlberg, R. G., Richer, H. B., McConnachie, A. W., et al. 2011, ApJ, 731, 124

Carretta, E., Bragaglia, A., Gratton, R. G., et al. 2009, A\&A, 505, 117

Catelan, M. 2009, Ap\&SS, 320, 261

Clement, C. M., \& Rowe, J. 2000, AJ, 120, 2579

Clementini, G. 2010, in Variable Stars, the Galactic halo and Galaxy

Formation, ed. C. Sterken, N. Samus, \& L. Szabados (Moscow: Sternberg

Astronomical Institute of Moscow Univ.), 107

Clementini, G., Di Tomaso, S., Di Fabrizio, L., et al. 2000, AJ, 120, 2054

Clementini, G., Gratton, R., Bragaglia, et al. 2003, AJ, 125, 1309

Collins, M. L. M., Chapman, S. C., Rich, R. M., et al. 2013, ApJ, 768, 172

Collins, M. L. M., Chapman, S. C., Rich, R. M., et al. 2014, ApJ, 783, 7

Conn, A. R., Ibata, R. A., Lewis, G. F., et al. 2012, ApJ, 758, 11

Cusano, F., Clementini, G., Garofalo, A., et al. 2013, ApJ, 779, 7

Cusano, F., Garofalo, A., Clementini, G., et al. 2015, ApJ, 806, 200

Cusano, F., Garofalo, A., Clementini, G., et al. 2016, ApJ, 829, 26

Gratton, R. G., Bragaglia, A., Clementini, G., et al. 2004, A\&A, 421, 937

Harris, W. E. 1996, AJ, 112, 1487

Ibata, R. A., Lewis, G. F., Conn, A. R., et al. 2013, Natur, 493, 62

Ibata, R. A., Lewis, G. F., McConnachie, A. W., et al. 2014, ApJ, 780, 128

Jacyszyn-Dobrzeniecka, A. M., Skowron, D. M., Mróz, P., et al. 2016, AcA, 66, 149
Jeffery, E. J., Smith, E., Brown, T. M., et al. 2011, AJ, 141, 171

Jurcsik, J., \& Kovacs, G. 1996, A\&A, 312, 111

Kapakos, E., Hatzidimitriou, D., \& Soszyński, I. 2011, MNRAS, 415, 1366

Kerp, J., Kalberla, P. M. W., Ben Bekhti, N., et al. 2016, A\&A, 589A, 120

Marconi, M., Coppola, G., Bono, G., et al. 2015, ApJ, 808, 50

Marconi, M., Fiorentino, G., \& Caputo, F. 2004, A\&A, 417, 1101

Marigo, P., Girardi, L., Bressan, A., et al. 2017, ApJ, 835, 77

Martin, N. F., Ibata, R. A., Lewis, G. F., et al. 2016, arXiv:1610.01158

Martin, N. F., Ibata, R. A., McConnachie, A. W., et al. 2013, ApJ, 776, 80

Monelli, M., Martínez-Vázquez, C. E., Bernard, E. J., et al. 2016, ApJ, 819,147

Oosterhoff, P. T. 1939, Obs, 62, 104

Pawlowski, M. S., Kroupa, P., Jerjen, H., et al. 2013, MNRAS, 435, 1928

Piersimoni, A. M., Bono, G., \& Ripepi, V. 2002, AJ, 124, 1528

Pritzl, B. J., Armandroff, T. E., Jacoby, G. H., \& Da Costa, G. S. 2002, AJ, 124, 1464

Pritzl, B. J., Armandroff, T. E., Jacoby, G. H., \& Da Costa, G. S. 2004, AJ, 127,318

Pritzl, B. J., Armandroff, T. E., Jacoby, G. H., \& Da Costa, G. S. 2005, AJ, 129,2232

Richardson, J. C., Irwin, M. J., McConnachie, A. W., et al. 2011, ApJ, 732 76

Ripepi, V., Marconi, M., Moretti, M. I., et al. 2014, MNRAS, 437, 2307

Sarajedini, A., Mancone, C. L., Lauer, T. R., et al. 2009, AJ, 138, 184

Schlegel, D. J., Finkbeiner, D. P., \& Davis, M. 1998, ApJ, 500, 525

Simon, N. R., \& Teays, T. J. 1982, ApJ, 261, 586

Stetson, P. B. 1987, PASP, 99, 191

Stetson, P. B. 1994, PASP, 106, 250

Stierwalt, S., Liss, S. E., Johnson, K. E., et al. 2017, NatAs, 1, 0025

Veljanoski, J., Mackey, A. D., Ferguson, A. M. N., et al. 2014, MNRAS, 442, 2929

Yang, S.-C., \& Sarajedini, A. 2012, MNRAS, 419, 1362 